\title{
Receiver-Based Channel Allocation in Cognitive Radio Wireless Mesh Networks
}

\author{
Hisham M. Almasaeid and Ahmed E. Kamal
}

\begin{abstract}
In this paper, we study the channel allocation problem in cognitive radio wireless mesh networks (CR-WMNs). We aim at finding an allocation strategy that guarantees QoS (link reliability), maximizes network coverage, and alleviates the need for a common control channel to coordinate the communication process. The allocation of a particular channel to a mesh client (MC) is considered feasible if the MC can establish connectivity with the backbone network in both the upstream and the downstream directions, and has the SINR (signal-to-interference and noise-ratio) of the uplink and the downlink with its parent mesh router (MRs) within a predetermined threshold. A receiver-based channel allocation (RBA) model that achieves the aforementioned objectives is proposed (channel assignment under this model can be proven to be NP-hard). We then formulate a mixed integer linear program, of the channel allocation problem under the proposed model, and compare its performance to that of two other baseline models, namely, transmitter-based and all-tunable channel allocation strategies. The results prove the superiority of the proposed model. We also developed a heuristic algorithm, which is shown to be an accurate algorithm.
\end{abstract}

\section{INTRODUCTION}

Motivated by the significant variance in channel utilization both spatially and temporally, a new dynamic spectrum access/allocation communication paradigm has recently been devised [1]. Empowered by the emerging technology of cognitive radios [2], the new paradigm allows unlicensed wireless users (usually referred to as secondary users (SUs)) to opportunistically access portions of the spectrum that are licensed to some other users (usually referred to as primary users (PUs)) but are currently unused (vacant). However, whenever a PU is back to use its licensed channel(s), all SUs that are currently using this channel must vacate to avoid interference with the primary network. This new paradigm solves the problems of spectrum underutilization and crowdedness associated with the current fixed channel allocation policy adopted by FCC [1].

Although its potential appears promising, cognitive radio networking entails several challenges that are not present in traditional wireless networks. Such challenges include spectrum sensing, allocation, management, and sharing in addition to network coordination and some upper-layer issues.

The cognitive radio technology allows SUs to change their communication parameters like power, operating frequency, and modulation dynamically. Although it gives SUs more flexibility and adaptability, it makes the coordination of the communication process much more complicated. This complexity arises from the fact that SUs might be operating on different frequency channels at different times. This requires the communicating pair of SUs to negotiate their channel availability and decide on one channel for communication. But, the negotiation itself must take place over a common channel that is known to the communicating pair apriori; this channel is usually referred to as the common control channel $(C C C)$, and it must be common network wide to guarantee network operation. Relying on a CCC has drawbacks, like:

(1) Depending on the network size (area and number of nodes), the number and distribution of primary stations, and the pattern of primary channels usage, the probability of having a CCC might be very low [3].

(2) A denial of service (DoS) attack that jams the CCC will break the operation of the entire network.

(3) Sharing one control channel between all SUs may lead to congestion on this channel which will consequently cause performance degradation for the overall network.

A number of alternatives to the $C C C$ approach have been introduced recently, some of them are reviewed next.

a) Local control channels: Instead of a single control channel common to all SUs [4], Zhao et al. [3] proposed the use of local control channels (LCCs) each of which is common to only a group of SUs. Using this approach, SUs at group boundaries, i.e., those that have neighbors in two different groups, might have to use (listen to and transmit on) more than one control channel. Although this approach is better than the CCC approach, it has its own drawbacks. First, the jamming problem is not alleviated although the scale of its effect is reduced to a group-level rather than a network-level. Second, SUs at group boundaries need to be either equipped with multiple transceivers or keep switching a single transceiver to listen to multiple LCCs, as well as the data channel.

b) Selective broadcasting: another alternative solution was to broadcast control information either over all available channels [5] or over a small subset of channels which covers all the neighbors of a node [6]. In [6], each node transmits the control information on a selected group of channels instead of a single control channel and this is why the approach is called selective broadcasting. Similar to the previous solution, a node might have to listen to more than one control channel. This requires listening and transmission operations to be synchronized for successful exchange of control information.

In this paper, we study the problem of channel allocation with QoS guarantees in CR-WMNs [7] assuming that both MCs and MRs employ cognitive radios and have to exploit unused licensed channels. The objective is to devise a channel allocation strategy that simplifies the coordination function of CR-WMNs and maximizes the number of served MCs.

\section{A. Related work}

Our work jointly addresses several operational aspects of cognitive radio networks. Those are:

1) Routing: we need to establish routes to connect every serviced MC to at least one of the gateway mesh routers via the mesh router servicing the cell of that MC. 
2) Common Control Channel (CCC): as CCC is one of the operational requirements of CR networks, we have to address in this paper. We will show that the proposed RBA strategy alleviates the need for a CCC.

3) Channel Assignment: we also address the problem of assigning data channels to CR nodes (MCs and MRs).

4) Power Control and Network Coverage: to maximize the coverage of the CR-WMN, we need to control the transmission power on both uplinks and downlinks.

In this section, we review the literature for the work addressing each of the aforementioned aspects.

1) Routing: Due to the opportunistic nature of spectrum access in CRNs, routing protocols developed for traditional wireless networks were not suitable and new protocols were needed. Routing protocols developed for CRNs are usually developed under either quasi-static channel state or dynamic channel state. In quasi-static channels, the state of a channel (idle or occupied by PUs) does not change for a relatively long period of time. In dynamic channels, on the other hand, the state of a channel varies over relatively short and random periods of time. It is somehow affordable to use centralized routing algorithms in the case of quasi-static channels (e.g., [8], [9], [10], [11]), but decentralized techniques are more suitable and more commonly used (e.g., [12], [13], [14], [15]) in the other case. The main difference between the routing problem in this paper and those previously proposed in literature is the directionality of routes. Under the proposed receiver-based channel allocation strategy, a route that is feasible from node $A$ to node $B$ is not necessarily feasible in the reverse direction (i.e., from $B$ to $A$ ). However, in existing routing approaches for CRNs, a route is almost always assume to work in both directions. See [16] for more information.

2) The Common Control Channel (CCC) problem: Another dimension we consider in this paper is the need for a CCC to coordinate the communication process. This problem has been the subject of a large number of research papers, recently surveyed in [17]. As classified in [17], four major schemes were used to implement control channels:

1) Sequence-based ([18], [19]): where the selection of control channels follows a random or predetermined channel hopping sequence. This scheme suffers from two major issues. The hopping sequence must adapt to the activity of PUs, which is difficult, and this approach can't support multicast of control information.

2) Group-based ([20], [21]): in which a group of nodes, close to each other, share a CCC. Then, some nodes need to act as bridges between adjacent groups, which must have the control channels of both nodes available. This scheme is challenging in terms of constructing the groups, selecting channels, managing inter-group communication, and group maintenance.

3) Dedicated ([22], [23]): which requires the selection of a predetermined channel as a CCC, which can be in the licensed or unlicensed bands. The existence of such a channel and its potential saturation is a challenge. Also, attacking this channel will affect the entire network.

In this paper, we alleviate the need for a CCC all together by introducing a receiver-based channel assignment strategy.
3) Channel Assignment: The problem of channel assignment in WMNs has been dealt with extensively in the literature. Reference [24] discusses the challenges in channel assignment in such networks, and surveys a number of approaches. The hybrid approach developed within the Net$\mathrm{X}$ framework [25] is the closest to our proposed approach. However, it is developed for the case of nodes equipped with multiple interfaces; or, when a single interface is used, it is fixed on the same channel for both transmission and reception, and special nodes with multiple interfaces are used to achieve connectivity. Reference [26] looks in particular at the problem of channel assignment in such networks with multiple radios.

References [27], [28] proposed routing and link layer protocols for multi-channel multi-interface ad hoc wireless networks. These works are different from ours in a number of ways. They tackle an ad hoc network, whereas we address a mesh network. They also assume the availability of all channels at all nodes, which is not the case in our work. Finally, in our network scenario, the channel assignments are made to guarantee upstream connectivity (from an MC to a gateway via MRs), and downstream connectivity (from a gateway to an MC via MRs), which is different from connectivity requirements in [27], [28].

The recent comprehensive survey in [29] considers different WMNs' design approaches, including channel assignment strategies. Most static channel assignment algorithms, e.g., [30], [31], [32], [33], [34], [35] use multiple radio interfaces per node to achieve connectivity. Most approaches also use graph coloring to assign radios to interfaces in order to avoid conflicts. Several of these approaches also consider the joint channel assignment and power allocation, e.g., [36], [37].

All of the above approaches were developed for WMNs at large, and do not take into consideration the properties of CRNs, and in particular the spatial channel heterogeneity property, i.e., having SUs observe different sets of available channels. Depending on the geographical distribution of SUs and PUs and the channel usage distribution of PUs, different SUs may observe different sets of available channels. Therefore, improper channel assignment may break network connectivity. Thus, any channel assignment algorithm designed for CRNs must take network connectivity into account. This problem is not present in traditional multi-channel wireless networks as all nodes share the same pool of channels.

4) Power Control and Network Coverage: Power control is an important dimension in many research papers in the area of CRNs. It might be addressed for different objectives. The objective in [38] was to assign channels to downlinks such that the interference at PUs is bounded and the downlink reliability is above a predefined threshold. Our work, however, considers downlink, uplink and network connectivity all together.

The problem of channel selection in multihop CR-WMNs was studied in [39]. The main objective was to select a channel for a node to transmit on such that the interference temperature ${ }^{1}$ within its transmission range does not exceed a predefined threshold.

\footnotetext{
${ }^{1}$ A new metric proposed by FCC that accounts for the cumulative radio frequency energy from transmissions and sets a maximum limit on it.
} 
Maximizing the number of SUs served on the PU licensed spectrum was the objective of controlling the transmission power of SUs in [40]. In [41], power control was studied jointly with spectrum allocation to maximize throughput.

These works share similarities with ours regarding power control. However, the differences can be summarized as:

(1) Our work combines power control problem with other issues including connectivity with gateways (upstream and downstream) and uplink/downlink reliability.

(2) Some of those works control the power to protect PUs, so they don't use the notion of available channels in the strict sense. Rather, they assume that all channels are available as long as the accumulated interference at PUs is not harmful. In our work, we deal only with channels, and thus the power control accounts for the interference between SUs.

\section{B. Paper Contributions}

The contributions of this work are as follows:

- We propose a receiver-based channel allocation model where each node receives on a fixed channel, while having the flexibility to transmit on any of the available channels. This model has two advantages. First, it does not require a CCC to coordinate the communication process. Second, it achieves better network coverage as the saved control channel can be used for data transmission.

- We present mixed integer linear program (MILP) formulations for the proposed channel allocation model, as well as for the baseline models we used for comparison.

- We present a heuristic algorithm to solve the problem.

\section{Paper Organization}

The rest of this paper is organized as follows. In Section II, we present the network model and layout the assumptions of this work. The receiver-based channel allocation (RBA) strategy is presented in Section III. In Section IV, we study the complexity of the receiver-based channel allocation problem and propose a mixed integer linear program (MILP) formulations for different channel allocation strategies. In Section $\mathrm{V}$, we propose a heuristic algorithm for the receiver-based channel allocation problem in CR-WMNs. The performance of the proposed heuristic algorithm is evaluated in Section VII. We also evaluate the optimal performance of the proposed RBA strategy versus other possible allocation strategies in Section VII. We conclude the paper in Section VIII.

\section{System ModeL}

In this section, we present the system model and assumptions and state the objectives of this work.

\section{A. Assumptions}

The general Cognitive Radio Wireless Mesh Network (CRWMN) structure consists of a number of MRs, some of them are directly connected to a backbone network, each of which manages all the MCs in its cell. Furthermore,

- Each MC is associated with exactly one MR, and communication within the cell takes place in one hop.

- All MCs and MRs are equipped with cognitive radios, and they communicate with each other through wireless communication over the unused licensed channels to reach the backbone network.

- We assume that a subset of MRs, that we call gateways, are directly connected to the backbone network. Therefore, each non-gateway MR should be able to reach at least one of the gateways in multiple hops of MRs in order to establish connectivity with the backbone network. From now on, we use the word gateway to refer to an MR that is directly connected to the backbone network, and use the abbreviation $M R$ to refer to a mesh router regardless of whether it is a gateway or not, and use the word node to refer to an SU (MR or MC).

- For each served MC, the reliability of its uplink $(M C \rightarrow$ $M R)$ and that of its downlink $(M R \rightarrow M C)$ must meet a given QoS requirement (a threshold reliability).

Throughout this paper, we assume the following:

- The channel availability at an SU is quasi-static (i.e., does not change in a short period of time). Thus, this work is more suited to spatial spectrum underutilization than temporal underutilization. However, it can still be used for the case of temporal spectrum underutilization if the PU activity is not very dynamic. Also, the channels are assumed to be orthogonal to each other.

- A simple path loss model for channel attenuation.

- The channel noise power is assumed to be the same at all locations on all channels. Furthermore, all cognitive radios are assume to have the same transceiver sensitivity.

- A dissemination protocol is used to transfer the calculated channel assignment to different nodes in the network. Flooding can be suitable in this case, given our assumption that channels are quasi-static.

Spectrum Sensing: In this work, we assume that the cognitive mesh network rely on a Spectrum Sensing and Management Entity (SSME) which provides unlicensed users with information about spectrum occupancy. The SSME can be any of the existing approaches like [42], [43], [44].

\section{B. Objective}

Our objective now is to evaluate the performance of different channel allocation strategies for cognitive radio mesh networks. For any allocation strategy to be feasible, the following two conditions must be satisfied for all served MCs.

(1) A path from the MC to at least one gateway must exist; we call this the upstream connectivity constraint. Also, a path from at least one gateway to the MC must exist; we call this the downstream connectivity constraint. The two paths may be disjoint. Also the upstream and downstream gateways may be different.

(2) Potential interference caused by intra-cell communication from cells other than the parent cell of an MC must be bounded to achieve a predetermined SINR to guarantee a BER (bit error rate) QoS requirement.

We aim at finding an allocation strategy that satisfies the above two conditions for the maximum number of MCs.

\section{Motivation And Challenges}

Based on the joint temporal and spatial distribution of the availability of the licensed spectrum, different SUs might 


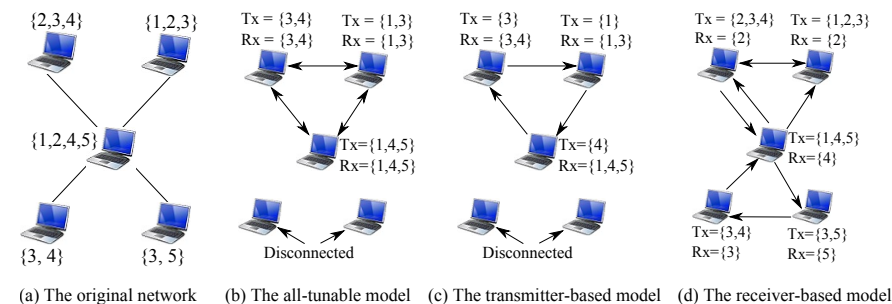

Fig. 1. An example to illustrate the motivation behind the proposed receiverbased channel allocation model. The number inside the black circle indicates the channel used for communication on the corresponding directional link.

observe different sets of available channels. Therefore, four modes of operation can be defined for each node's transceiver:

1) Tunable Transmitter - Tunable Receiver (TT-TR): an SU can transmit/receive on any of the available channels.

2) Tunable Transmitter - Fixed Receiver (TT-FR): an SU can transmit on any of the available channels, but receives on a fixed channel.

3) Fixed Transmitter - Tunable Receiver (FT-TR): an SU can receive on any of the available channels, but transmits on a fixed channel.

4) Fixed Transmitter - Fixed Receiver (FT-FR): an SU transmits/receives on a fixed channel.

TT-TR is the most commonly assumed communication paradigm in multihop cognitive radio networks. It allows an SU to use any of its available channels for transmission and/or reception. Therefore, the channel allocation problem under this paradigm will be to assign channels to links. This means that a node might use different channels for its incoming and outgoing communication links with its neighbors. The drawback of this paradigm, which is shared with the FT-TR paradigm, is that a CCC is needed for channel negotiation. In other words, these two communication paradigms cannot be used without a CCC because the transmitter needs to inform the receiver about its intention to transmit so that the receiver can tune to the transmitter's channel. Because of the problems of the CCC approach discussed earlier in Section I, and the fact that the probability that a CCC exists could be low [3], it is a necessity to devise a new communication paradigm in which the requirement of a CCC is avoided. In this paper, we propose an allocation strategy based on TT-FR, we call this strategy a receiver-based channel allocation (RBA).

Definition 3.1: RBA: is a channel allocation strategy, based on the $T T-F R$ mode of operation, under which, each node is allocated a fixed channel for reception, but is allowed to transmit on any available channel.

Therefore, under RBA, if each node knows the channels allocated to its neighbors, no channel negotiation is needed for transmission coordination, and thus no CCC is needed for this purpose. Furthermore, we will consider two more allocation strategies, namely Transmitter-Based Allocation (TBA) and All-Tunable Allocation (ATA), these are defined next.

Definition 3.2: TBA: is a channel allocation strategy under which, each node is allocated a fixed channel for transmission, but is allowed to receive on any of the available channels. Therefore, it is based on the $F T-T R$ mode of operation.

Definition 3.3: ATA: is a channel allocation strategy under which, each node is allowed to tune to any available channel
TABLE I

DIFFERENCES BETWEEN THE RBA, TBA, AND ATA CHANNEL ALLOCATION STRATEGIES.

\begin{tabular}{l|c|c|c|c|c|c}
\hline \hline & \multicolumn{2}{|c|}{ RBA } & \multicolumn{2}{c|}{ TBA } & \multicolumn{2}{c}{ ATA } \\
\hline & Rx Ch. & Tx Ch. & Rx Ch. & Tx Ch. & Rx Ch. & Tx Ch. \\
\hline MR & fixed & tunable & tunable & fixed & tunable & tunable \\
\hline MC & fixed & fixed & fixed & fixed & fixed & fixed \\
\hline CCC & \multicolumn{2}{|c|}{ No } & \multicolumn{2}{c}{ Yes } & \multicolumn{2}{c}{ Yes } \\
\hline
\end{tabular}

for either reception or transmission. Therefore, it is based on the $T T-T R$ mode of operation.

Table I summarizes the differences between all strategies.

\section{A. Issues and challenges}

In this subsection, we address some issues and challenges related to the proposed RBA approach.

a) Degree of connectivity: it is expected that the assignment of one channel for each node to receive on will result in a decreased level of connectivity. One might think that the TT-TR approach will result in the highest degree of connectivity, because transmission and reception are allowed on any channel, which intuitively implies that the number of served MCs will be higher compared to TT-FR. However, this is not necessarily true because of the following:

- The TT-TR approach requires the existence of a CCC, while the TT-FR approach does not, and the probability that the network is connected depends on the probability that a CCC exists.

- The channel used as a CCC cannot be used for data communication. This gives the TT-FR one more channel to use for data communication.

- The effect on connectivity depends on the channel availability distribution at a node and its neighbors. It is more likely for a channel available at a particular node to be available at its neighbors, which reduces the likelihood that a node gets disconnected from its neighbors when it is assigned a fixed channel to receive on.

Consider the example in Figure 1. It shows a network of five nodes exploiting cognitive radios. Under both ATA and TBA models, channel 2 is selected as the common control channel as it connects the maximum number of nodes, and that is 3 . Therefore, connectivity can be established between three nodes only as shown in Figures 1.(b) and 1.(c). However, when RBA is used, connectivity is established between the five nodes as there is no need for a common control channel (Figure 1.(c)).

b) Deafness Problem: the deafness problem is recognized in wireless networks with directional antennas [45]. Deafness is caused when node $A$ wants to communicate with node $B$ while $B$ is currently communicating with node $C$. Node $A$ interprets the absence of a reply from $B$ (caused by the fact that $B$ 's antenna is tuned to the direction of node $C$ ) as a collision at $B$, and consequently backs-off ${ }^{2}$. The problem becomes worse if $B$ has multiple packets to transmit, which will cause $A$ to unnecessarily back-off several times. The same problem may occur under the proposed RBA approach. Let $f_{A}, f_{B}$, and $f_{C}$ be the frequency channels assigned to nodes $A, B$, and $C$ for reception respectively. Assume that node $A$ wants to communicate with node $B$

\footnotetext{
${ }^{2}$ This is based on CSMA/CA medium access.
} 
which is currently communicating with node $C$ on channel $f_{C}$. Then, $A$ will fail to reach $B$ on $f_{B}$ because $B$ is currently tuned to $f_{C}$, and node $A$ unnecessarily backs-off, which results in the same deafness problem recognized in the directional antennas case. This problem is not present in TT-TR and FT-TR because the neighbors of the transmitter will know about the ongoing communication by overhearing the control information transmitted over the CCC.

c) Multi-channel hidden node problem: the last challenge is the hidden node problem. This problem is well known in wireless communication, however, it is a bit different using the $T T-F R$ approach. In traditional single-channel wireless networks, under an IEEE 802.11 based MAC protocol, an RTS/CTS handshake between the transmitter and the receiver solves the hidden node problem. However, following the TT$F R$ mode, hidden nodes may exist on multiple channels. This means that the RTS/CTS handshake must be cloned on multiple channels, which results in significant delay if a single radio interface is used.

d) Unidirectional routes: Under the RBA strategy, a route from $A$ to $B$ may not be feasible in the reverse direction (i.e., from $B$ to $A$ ). To illustrate this issue, consider the to the example in Figure 2. The part to the left shows five mesh routers (represented by circles), named $s$ (source), $r_{0}-r_{2}$ (intermediate relays), and the Gateway mesh router (the one that has access to the Internet). The figure also shows 5 primary users (represented by squares) which are using four channels each was given a unique color. The colored dashed circle around each primary user shows its transmission range. Assume that the transmission range of the mesh routers equals that of primary users. Now assume that we assign channels (for reception only, i.e., following the receiver based allocation model) to mesh routers as follows: Gateway is assigned Channel Ch. 1, $r_{2}$ is assigned Ch. 1, $r_{1}$ is assigned Ch. 4, $r_{0}$ is assigned Ch. 3, and $s$ is assigned Ch. 3.

This assignment guarantees upstream connectivity, i.e., from $s$ to the Gateway because the following transmission can be done without harming PUs: $s$ transmits on Ch. 3, $r_{0}$ on Ch. 4, $r_{1}$ on Ch. 1, and $r_{2}$ on Ch. 1. However, it does not guarantee downstream connectivity because the transmissions marked with a red cross will harm PUs.

\section{MILP FORMULATIONS}

Before we give a formal definition for the RBA problem in CR-WMNs, we present some notations and terminology.

- $\mathcal{B}$ is the set of non-gateway MRs .

- $\mathcal{G}$ is the set of gateways.

- $\mathcal{A}_{i}$ is the set of MCs that belong to the cell administrated by MR $i$. $\mathcal{A}$ is the set of all MCs in the network, i.e., $\mathcal{A}=\bigcup_{i \in \mathcal{B} \cup \mathcal{G}} \mathcal{A}_{i}$.

- $\mathcal{L}$ is the set of all channels in the system, where $|\mathcal{L}|=K$.

- $\mathcal{L}_{i}$ is the set of available channels at node $i$. A channel is said to be available to an MR if it is idle (i.e., identified as unused by the SSME). On the other hand, a channel is said to be available to an MC if it is available to the MR managing the cell of this $\mathrm{MC}^{3}$, and it is identified

\footnotetext{
${ }^{3} \mathrm{An} \mathrm{MC}$ cannot make use of a channel that is not available at its parent MR to communicate with that MR
}

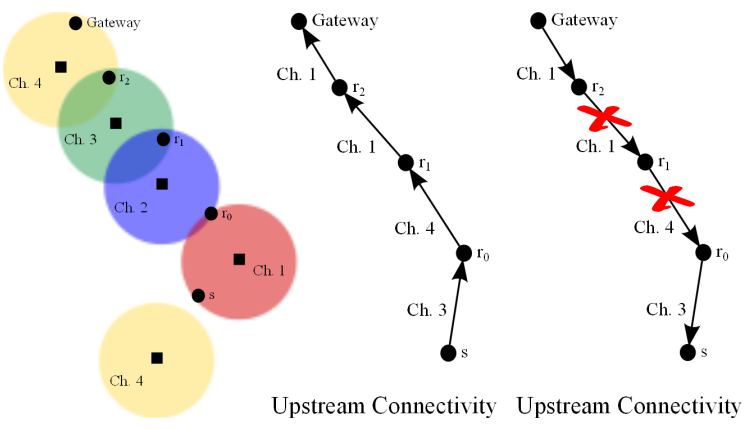

Fig. 2. An example to illustrate unidirectionality of routes under RBA.

idle by the SSME at the MC.

- $P_{i}^{k}$ is the transmission power of node $i$ on channel $k$ such that $P_{i}^{k} \leq P_{r}^{\max } \forall i \in \mathcal{B} \cup \mathcal{G}$, and $P_{i}^{k} \leq P_{c}^{\max } \forall i \in \mathcal{A}$. $P_{r}^{\max }$ and $P_{c}^{\max }$ are the maximum transmission powers of an MR and an MC respectively.

- $\Psi_{i j}^{k}$ is the channel power gain from $i$ to $j$ on channel $k$.

- $\zeta_{i j}^{k}$ is the maximum amount of interference that the cell managed by MR $i$ may produce at the location of node $j$ on channel $k$.

$\zeta_{i j}^{k}=\max \left\{\max _{w \in \mathcal{A}_{i}} P_{w}^{k} \Psi_{w j}^{k}, P_{i}^{k} \Psi_{i j}^{k}\right\}$

- $\zeta_{i j}^{\max }$ is the maximum amount of interference that the cell managed by MR $i$ produces at the location of node $j$ at maximum transmission power, i.e.,

$$
\zeta_{i j}^{\max }=\max \left\{\max _{w \in \mathcal{A}_{i}} \max _{k \in \mathcal{L}_{w}} P_{c}^{\max } \Psi_{w j}^{k}, \max _{k \in \mathcal{L}_{i}} P_{r}^{\max } \Psi_{i j}^{k}\right\}
$$

- $N_{0}$ is the channel noise power, and it is assumed to be the same at all locations on all channels.

- $\gamma$ is the minimum SINR value required to guarantee a certain BER at a node (reliability threshold).

- $c_{j}^{k}$ is a binary variable that is set to 1 if channel $k$ is assigned to node $j$, and 0 otherwise.

\section{A. Receiver-based channel allocation (RBA) problem}

The receiver-based channel allocation (RBA) problem in wireless cognitive mesh networks is defined as follows:

Definition 4.1: RBA problem: given a wireless cognitive mesh network of $\mathcal{G}$ gateway MRs, $\mathcal{B}$ non-gateway MRs, and $\mathcal{A}_{i}$ MCs managed by MR $i$ for all $i \in \mathcal{B} \cup \mathcal{G}$. Also, for all $j \in \mathcal{B} \cup \mathcal{G} \cup \mathcal{A}$, the geographic location of $j$ and its channel availability $\mathcal{L}_{j}$ are given. Find a $T T-F R$ channel allocation that maximizes the number of served MCs such that for each served $M C$, the following conditions are satisfied:

1) A path from each MC (through its parent MR) to at least one $M R$ in $\mathcal{G}$ exists.

2) A path from at least one $M R$ in $\mathcal{G}$ to each $M C$ (through its parent $M R$ ) exists.

3) The SINR of the uplinks ( $M C \rightarrow M R$ ) and the downlinks $(M R \rightarrow M C)$ is at least $\gamma$.

Note that the upstream and downstream paths for an MC must go through its parent MR. Therefore, the RBA problem can be decomposed into two subproblems: (1) channel allocation to MRs such that the upstream/downstream connectivity constrain is satisfied for MRs. (2) channel allocation to MCs such that reliable uplinks/downlinks with MRs are established 
for the maximum number of MCs. The first subproblem can be represented as a network flow formulation as we show throughout this subsection. By adding few more constraints to jointly model the second subproblem, the whole RBA problem can then be formulated as an MILP.

To show the hardness of the RBA problem, let us just consider the upstream/downstream connectivity subproblem.

Definition 4.2: Upstream/downstream connectivity problem (UDCP): given a network graph $G(\mathcal{B} \cup \mathcal{G}, E)$, where $E$ is the set of connectivity edges between MRs. If the channel availability at each $M R\left(\mathcal{L}_{i} \forall i \in \mathcal{B} \cup \mathcal{G}\right)$ is known, is there a receiver-based channel assignment that guarantees upstream and downstream connectivity for each non-gateway MR?

It can be shown that the UDCP problem is NP-Complete (and its optimization version is NP-hard) by a reduction from the maximum satisfiability problem ${ }^{4}$. As UDCP is a subproblem of the RBA problem, then the RBA problem is at least as hard as the UDCP problem.

We start with the network flow formulation for the first subproblem, i.e., upstream/downstream connectivity. Define a graph $G=(V, E \cup \bar{E})$ of a set of vertices $V=\mathcal{B} \cup \mathcal{G} \cup$ $\{s, \bar{s}, d, \bar{d}\}$ and a set of edges $E \cup \bar{E}$. The vertices $s$ and $d$ represent a hypothetical source and hypothetical sink for the upstream flow respectively. On the other hand, $\bar{s}$ and $\bar{d}$ represent hypothetical source and sink for the downstream flow respectively. $E / \bar{E}$ are the sets of upstream/downstream edges respectively. $E$ is defined as follows:

- A directed edge $e=(s, j)$ exists for each vertex $j \in$ $\mathcal{B} \cup \mathcal{G}$. The flow on such an edge is equal to the number of served MCs that belong to $\mathcal{A}_{j}$, i.e., $\sum_{i \in \mathcal{A}_{j}} \sum_{k \in \mathcal{L}_{i}} c_{i}^{k}$.

- A directed edge $e=(i, d)$ exists for each vertex $i \in \mathcal{G}$.

- A directed edge $e=(i, j)$ exists for any pair of MRs $i, j \in \mathcal{B} \cup \mathcal{G}$ if $\exists k \in \mathcal{L}_{i} \cap \mathcal{L}_{j}$ such that,

$\Psi_{i j}^{k} P_{r}^{\max } \geq P_{t h}$

where $P_{t h}$ is a threshold received signal strength requirement to detect the transmission. The capacity of such an edge is given as $|\mathcal{A}| \cdot \sum_{k \in \mathcal{L}_{i} \cap \mathcal{L}_{j}} c_{j}^{k}$, i.e., the end-node $j$ must be assigned a channel that belongs to the list of available channels at the start-node $i$ in order for a flow upper-bounded by $|\mathcal{A}|$ to pass through the edge $e$.

On the other hand, the set $\bar{E}$ is defined as follows:

- A directed edge $e=(\bar{s}, j)$ exists for each vertex $j \in \mathcal{G}$.

- A directed edge $e=(i, \bar{d})$ exists for each vertex $i \in \mathcal{B} \cup \mathcal{G}$. The flow on such an edge is equal to the number of served MCs that belong to $\mathcal{A}_{i}$, i.e., $\sum_{j \in \mathcal{A}_{i}} \sum_{k \in \mathcal{L}_{j}} c_{j}^{k}$.

- A directed edge $e=(i, j)$ exists for any pair of MRs $i, j \in \mathcal{B} \cup \mathcal{G}$ if condition (3) is satisfied for at least one channel $k \in \mathcal{L}_{i} \cap \mathcal{L}_{j}$. The capacity of such an edge is given as $|\mathcal{A}| \cdot \sum_{k \in \mathcal{L}_{i} \cap \mathcal{L}_{j}} c_{j}^{k}$.

We define two flow commodities, one for the upstream flow (through the edges in $E$ ) and one for the downstream flow (through the edges on $\bar{E}$. Let $f_{i j}$ denote the flow on edge $(i, j) \in E$, i.e. upstream flow, and $g_{i j}$ denote the flow on edge $(i, j) \in \bar{E}$, i.e. downstream flow. The network flow representation is shown in Figure 3. We now consider

\footnotetext{
${ }^{4}$ The proof is included in a supplementary document
}

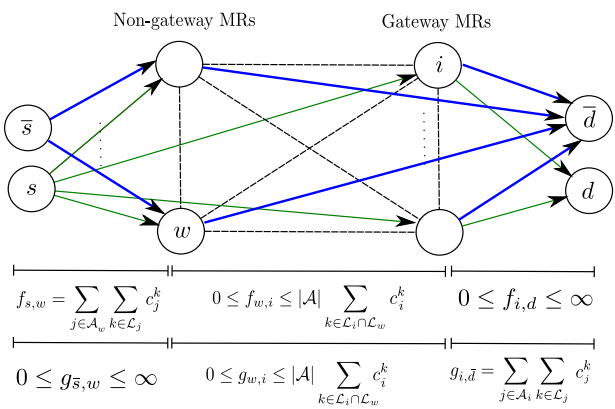

Fig. 3. The network flow representation of the upstream and the downstream connectivity. Bounds on the flow on the three groups of edges (source-to-MR, MR-to-MRs, and MR-to-destination) are shown below the graph drawing.

the second subproblem we mentioned earlier, i.e., channel allocation to MCs. It is of no benefit to assign a channel to an MC $j$ unless its parent MR is assigned one that is common between the two, otherwise, MC $j$ cannot be served. Thus,

$\sum_{k \in \mathcal{L}_{j}} c_{j}^{k} \leq \sum_{k \in \mathcal{L}_{i} \cap \mathcal{L}_{j}} c_{i}^{k}, \forall i \in \mathcal{B} \cup \mathcal{G}, j \in \mathcal{A}_{i}$

Then, the downlink (from an MR to an MC) reliability is achieved if the following inequality is satisfied:

$$
\begin{aligned}
& \Psi_{i j}^{k} P_{i}^{k}-\gamma\left(N_{0}+\sum_{m \in \mathcal{B} \cup \mathcal{G} \backslash\{i\}} \zeta_{m j}^{k}\right) \geq \\
& \gamma\left(c_{j}^{k}-1\right)\left(N_{0}+\sum_{m \in \mathcal{B} \cup \mathcal{G} \backslash\{i\}} \zeta_{m j}^{\max }\right), \\
& \forall i \in \mathcal{B} \cup \mathcal{G}, j \in \mathcal{A}_{i}, k \in \mathcal{L}_{i} \cap \mathcal{L}_{j}
\end{aligned}
$$

If $\mathrm{MC} j$ is assigned channel $k$, the right hand side becomes:

$$
\Psi_{i j}^{k} P_{i}^{k} \geq \gamma\left(N_{0}+\sum_{m \in \mathcal{B} \cup \mathcal{G} \backslash\{i\}} \zeta_{m j}^{k}\right)
$$

and $P_{i}^{k}$ must be set to a value that makes the received power $\left(\Psi_{i j}^{k} P_{i}^{k}\right)$ greater than or equal to the SINR threshold $(\gamma)$ at MC $j$ multiplied by the accumulated noise $\left(N_{0}+\right.$ $\sum_{m \in \mathcal{B} \cup \mathcal{G} \backslash\{i\}} \zeta_{m j}^{k}$ ). On the other hand, if MC $j$ is not assigned channel $k$, i.e., $c_{j}^{k}=0$, then the inequality becomes:

$$
\Psi_{i j}^{k} P_{i}^{k} \geq \gamma\left(N_{0}+\sum_{m \in \mathcal{B} \cup \mathcal{G} \backslash\{i\}} \zeta_{m j}^{k}\right)-\gamma\left(N_{0}+\sum_{m \in \mathcal{B} \cup \mathcal{G} \backslash\{i\}} \zeta_{m j}^{\max }\right)
$$

or equivalently:

$$
\Psi_{i j}^{k} P_{i}^{k} \geq \gamma \sum_{m \in \mathcal{B} \cup \mathcal{G} \backslash\{i\}} \zeta_{m j}^{k}-\zeta_{m j}^{\max }
$$

As $\zeta_{m j}^{\max }$ is an upper bound on $\zeta_{m j}^{k}$, the inequality will be satisfied in this case by any non-negative value of $P_{i}^{k}$.

Similarly, the uplink (from an MC to an MR) reliability is achieved if the following inequality is satisfied:

$$
\begin{aligned}
& \Psi_{j i}^{k} P_{j}^{k}-\gamma\left(N_{0}+\sum_{m \in \mathcal{B} \cup \mathcal{G} \backslash\{i\}} \zeta_{m i}^{k}\right) \geq \\
& \gamma\left(c_{i}^{k}+\sum_{w \in \mathcal{L}_{j}} c_{j}^{w}-2\right)\left(N_{0}+\sum_{m \in \mathcal{B} \cup \mathcal{G} \backslash\{i\}} \zeta_{m i}^{\max }\right), \\
& \forall i \in \mathcal{B} \cup \mathcal{G}, j \in \mathcal{A}_{i}, k \in \mathcal{L}_{i} \cap \mathcal{L}_{j}
\end{aligned}
$$

The right hand side is equal to 0 if MR $i$ assigned channel $k$ and the MC is assigned a channel. In this case:

$$
\Psi_{j i}^{k} P_{j}^{k} \geq \gamma\left(N_{0}+\sum_{m \in \mathcal{B} \cup \mathcal{G} \backslash\{i\}} \zeta_{m i}^{k}\right)
$$


Therefore, $P_{j}^{k}$ must be set to a value that satisfies the required SINR threshold $\gamma$ at MR $i$. Also, if $k$ is not assigned to MR $i$ and MC $j$ is not assigned a channel, the inequality becomes:

$$
\Psi_{j i}^{k} P_{j}^{k} \geq \gamma \sum_{m \in \mathcal{B} \cup \mathcal{G} \backslash\{i\}} \zeta_{m i}^{k}-\zeta_{m i}^{\max }
$$

which will, as mentioned before, be satisfied with any nonnegative value of $P_{j}^{k}$. The last case is when only the MR or the $\mathrm{MC}$ is assigned a channel, but not the other. In this case:

$$
\Psi_{j i}^{k} P_{j}^{k} \geq-\gamma N_{0}+\gamma \sum_{m \in \mathcal{B} \cup \mathcal{G} \backslash\{i\}} \zeta_{m i}^{k}-2 \cdot \zeta_{m i}^{\max }
$$

Again, the inequality above will be satisfied with any non-negative value of $P_{j}^{k}$. Finally, the $R B A$ problem can be formulated as an MILP as follows:

Maximize $\sum_{i \in \mathcal{A}} \sum_{k \in \mathcal{L}_{i}} c_{i}^{k}$, subject to:

(a) Channel assignment: this set guarantees the assignment of at most one channel to each node (MC or MR). It also guarantees that no $\mathrm{MC}$ is assigned a channel unless its parent MR is assigned one that is shared between the two.

$$
\begin{aligned}
& \sum_{k \in \mathcal{L}_{j}} c_{j}^{k} \leq 1, \forall j \in \mathcal{B} \cup \mathcal{G} \cup \mathcal{A} . \\
& \sum_{k \in \mathcal{L}_{j}} c_{j}^{k} \leq \sum_{k \in \mathcal{L}_{i} \cap \mathcal{L}_{j}} c_{i}^{k}, \forall i \in \mathcal{B} \cup \mathcal{G}, j \in \mathcal{A}_{i}
\end{aligned}
$$

(b) Upstream connectivity constraints: this set guarantees that each MC that has been assigned a channel can establish a forward path to at least one gateway (see Figure 3-left).

$$
\begin{aligned}
& \sum_{j:(i, j) \in E} f_{i j}-\sum_{j:(j, i) \in E} f_{j i}=0, i \in \mathcal{B} \cup \mathcal{G} . \\
& \sum_{j:(s, j) \in E} f_{s j}=\sum_{j:(j, d) \in E} f_{j d} \\
& f_{s j}=\sum_{i \in \mathcal{A}_{j}} \sum_{k \in \mathcal{L}_{i}} c_{i}^{k}, j \in \mathcal{B} \cup \mathcal{G} . \\
& f_{i j} \leq|\mathcal{A}| \cdot \sum_{k \in \mathcal{L}_{i} \cap \mathcal{L}_{j}} c_{j}^{k}, \quad(i, j) \in E .
\end{aligned}
$$

(c) Downstream connectivity constraints: this set guarantees that a path from at least one gateway MR can be established to each MC that has been assigned a channel (see Figure 3-right).

$$
\begin{aligned}
& \sum_{j:(i, j) \in \bar{E}} g_{i j}-\sum_{j:(j, i) \in \bar{E}} g_{j i}=0, i \in \mathcal{B} \cup \mathcal{G} . \\
& \sum_{j:(\bar{s}, j) \in \bar{E}} g_{\bar{s} j}=\sum_{j:(j, \bar{d}) \in \bar{E}} g_{j \bar{d}} \\
& g_{j \bar{d}}=\sum_{i \in \mathcal{A}_{j}} \sum_{k \in \mathcal{L}_{i}} c_{i}^{k}, j \in \mathcal{B} \cup \mathcal{G} . \\
& g_{i j} \leq|\mathcal{A}| \cdot \sum_{k \in \mathcal{L}_{i} \cap \mathcal{L}_{j}} c_{j}^{k}, \quad(i, j) \in \bar{E} .
\end{aligned}
$$

(d) Power control constraints: this set of constraints keeps the transmission power of any node that has not been assigned a channel at the zero level, and that of those which have been assigned channels below a maximum level.

$$
\begin{aligned}
& P_{i}^{k} \leq P_{r}^{\max } \cdot \sum_{j:\left\{j \in \mathcal{A}_{i}, k \in \mathcal{L}_{j}\right\}} c_{j}^{k}, \forall i \in \mathcal{B} \cup \mathcal{G}, k \in \mathcal{L}_{i} \\
& P_{i}^{k} \leq P_{r}^{\max }, \forall i \in \mathcal{B} \cup \mathcal{G}, k \in \mathcal{L}_{i} \\
& P_{j}^{k} \leq P_{c}^{\max } \cdot c_{i}^{k}, \quad \forall i \in \mathcal{B} \cup \mathcal{G}, j \in \mathcal{A}_{i}, k \in \mathcal{L}_{i} \cap \mathcal{L}_{j}
\end{aligned}
$$

(e) Inter-cell interference: this set defines the lower-bound on the interference at each node caused by other cells.

$$
\begin{gathered}
\zeta_{i j}^{k} \geq P_{i}^{k} \Psi_{i j}^{k}, \quad \forall i \in \mathcal{B} \cup \mathcal{G}, j \in \mathcal{A} \cup \mathcal{B} \cup \mathcal{G} \backslash\left(\mathcal{A}_{i} \cup\{i\}\right), \\
k \in \mathcal{L}_{i} \cap \mathcal{L}_{j} \\
\zeta_{i j}^{k} \geq P_{m}^{k} \Psi_{m j}^{k}, \quad \forall i \in \mathcal{B} \cup \mathcal{G}, j \in \mathcal{A} \cup \mathcal{B} \cup \mathcal{G} \backslash\left(\mathcal{A}_{i} \cup\{i\}\right), \\
m \in \mathcal{A}_{i}, k \in \mathcal{L}_{m} \cap \mathcal{L}_{j}
\end{gathered}
$$

(f) Link reliability constraints: this set guarantees both uplink and downlink reliability for each MC that has been assigned a channel. The following two equations are the same as equations (4) and (5), and are repeated here for clarity.

$\Psi_{i j}^{k} P_{i}^{k}-\gamma\left(N_{0}+\sum_{m \in \mathcal{B} \cup \mathcal{G} \backslash\{i\}} \zeta_{m j}^{k}\right) \geq$

$\gamma\left(c_{j}^{k}-1\right)\left(N_{0}+\sum_{m \in \mathcal{B} \cup \mathcal{G} \backslash\{i\}} \zeta_{m j}^{\max }\right)$,

$\forall i \in \mathcal{B} \cup \mathcal{G}, j \in \mathcal{A}_{i}, k \in \mathcal{L}_{i} \cap \mathcal{L}_{j}$

$\Psi_{j i}^{k} P_{j}^{k}-\gamma\left(N_{0}+\sum_{m \in \mathcal{B} \cup \mathcal{G} \backslash\{i\}} \zeta_{m i}^{k}\right) \geq$

$\gamma\left(c_{i}^{k}+\sum_{w \in \mathcal{L}_{j}} c_{j}^{w}-2\right)\left(N_{0}+\sum_{m \in \mathcal{B} \cup \mathcal{G} \backslash\{i\}} \zeta_{m i}^{\max }\right)$,

$\forall i \in \mathcal{B} \cup \mathcal{G}, j \in \mathcal{A}_{i}, k \in \mathcal{L}_{i} \cap \mathcal{L}_{j}$

\section{B. Transmitter-based channel allocation (TBA)}

We refer to the channel allocation strategy that follows the FT-TR mode as the transmitter-based channel allocation (TBA). In TBA, a node is assigned a fixed channel to transmit on, while it can receive on any available channel. Again, this strategy requires the existence of a CCC so that the transmitter can make the receiver tune to its channel. Thus, we study this strategy under two assumptions: first, a preassumed CCC exists; second, the existence of a CCC depends on channel availability. The TBA problem is defined similar to the $R B A$ problem except that the $F T-T R$ is used, not $T T-F R$. For the MILP formulation of the TBA problem, see [46].

\section{All-tunable channel allocation (ATA)}

Following the TT-TR mode, we propose the All-tunable channel allocation (ATA) strategy, under which channels are assigned to links rather than nodes. Therefore, an MR might have to listen/transmit on different channels. As for the MC, it will have to receive on one channel (the one assigned to the downlink), and transmit on one channel (the one assigned to the uplink). We also study this allocation strategy under two assumptions: first, a preassumed CCC exists; second, the existence of a CCC depends on channel availability and it is not preassumed. The ATA problem is defined similar to the RBA problem except that TT-TR mode is used. For the MILP formulation of the ATA problem, we refer the reader to [46], as it was omitted due to lack of space. 


\section{HEURISTIC SOlUTION FOR RBA}

Section VII below shows that the RBA approach outperforms other allocation strategies. Our optimal approach in Section IV, as suggested earlier, is suitable for cases with spatial channel heterogeneity. However, the optimal channel allocation approach may not be suitable for more dynamic environments, or cases with temporal channel heterogeneity, where channel availabilities change over a short time scale. We therefore introduce a polynomial time complexity heuristic for the RBA problem. When the channels availabilities, or channel conditions change with time, this heuristic can be rerun to revise the assignment. We solve the problem in three phases:

(1) Channel assignment to MRs: in this phase, MRs are assigned channels such that their upstream and downstream connectivity with the gateway(s) is maintained.

(2) Finding the maximum number of reliable uplinks: based on the channel assignment made in the first phase, and the channel availability at each MC, we assign transmission powers to MCs such that the number of reliable uplinks $(\mathrm{MC} \rightarrow \mathrm{MR})$ is maximized. This power assignment is obtained by the algorithm we propose in Subsection V-B.

(3) Channel assignment to MCs: to the MCs that have reliable uplinks after phase (2), we allocate channels and transmission powers such that the number of reliably served MCs is maximized. An MC is reliably served if the reliability of its uplink and downlink is above $\gamma$.

\section{A. Phase 1: Channel assignment to MRs}

The first phase in our solution to the RBA problem is to allocate channels to MRs such that the upstream and downstream connectivity with the backbone network is established for the maximum number of MRs. Before introducing the algorithm, we first give some definitions:

- $\mathbf{L}_{(\mathbf{t})}$ is an $N_{r} \times K$ matrix where $N_{r}$ is the total number of MRs, and $K$ is the total number of channels available in the system. This matrix represents channels that an MR can transmit on, hence the (t) is the subscript of $\mathbf{L}$. Thus, the $(i, k)^{t h}$ element of $\mathbf{L}_{(\mathbf{t})}$ is defined as,

$$
\mathbf{L}_{(\mathbf{t})}[i, k]=\left\{\begin{array}{cc}
1 & \text { if } k \in \mathcal{L}_{i} \\
0 & \text { otherwise }
\end{array}\right.
$$

- $\mathbf{L}_{(\mathbf{r})}$ is an $N_{r} \times K$ matrix that represents the channels that an MR can receive on. Although this matrix is initially the same as $\mathbf{L}_{(\mathbf{t})}$, it will become different when MRs are assigned channels to receive on as we will see later. The $(i, k)^{t h}$ element of $\mathbf{L}_{(\mathbf{r})}$ is initially defined as,

$\mathbf{L}_{(\mathbf{r})}[i, k]=\left\{\begin{array}{cc}1 & \text { if } k \in \mathcal{L}_{i} \\ 0 & \text { otherwise }\end{array}\right.$

- I is an $N_{r} \times N_{r}$ matrix that represents the accessibility between MRs. In other words,

$\mathbf{I}[m, n]=\left\{\begin{array}{l}1, \text { if } \exists k \in \mathcal{L}_{m} \cap \mathcal{L}_{n}: \Psi_{m n}^{k} P_{r}^{\max } \geq P_{t h} \\ 0, \text { otherwise }\end{array}\right.$

- W is an $N_{r} \times K$ matrix that represents the weights of assigning channels to MRs. The element $\mathbf{W}[i, k]$ is the weight of assigning channel $k$ to MR $i$ defined as the number of MCs in $\mathcal{A}_{i}$ that can access $i$ on channel $k$.
- $\mathbf{C}_{(u)}$ is a row-vector of length $N_{r}$ such that $\mathbf{C}_{(u)}[i]=1$ if there exists a directed path from MR $i$ to the gateway, and equals 0 otherwise. This connectivity is evaluated assuming that $i$ is assigned all the channels that have their values in the row-vector $\mathbf{L}_{(r)}[i, *]$ set to 1 .

- $\mathbf{C}_{(d)}$ is a row-vector of length $N_{r}$ such that $\mathbf{C}_{(d)}[i]=1$ if there exists a directed path from the gateway to MR $i$, and equals 0 otherwise. Similar to $\mathbf{C}_{(u)}$, this connectivity is evaluated assuming that $i$ is assigned all the channels with their values in the row-vector $\mathbf{L}_{(r)}[i, *]$ set to 1 .

- Define: $\operatorname{argmax}(f(x), g(x)):=\{x \mid \forall y: f(y) \leq$ $f(x)$, and if $f(y)=f(x)$ then $g(y) \leq g(x)\}$

The Routers Channel Allocation (RCA) algorithm is outlined in Algorithm 1. First, the matrices $\mathbf{L}_{(t)}$ and $\mathbf{L}_{(r)}$ are calculated using equations (23) and (24) respectively. These two matrices are then used to evaluate the upstream and downstream connectivity vectors $\mathbf{C}_{(u)}$ and $\mathbf{C}_{(d)}$ respectively (line 2). The algorithm, then, operates iteratively and selects one MR at each iteration for processing. The gateway is selected first, then MRs are selected in breadth first manner based on their connectivity with already processed MRs. Let MR $i$ be the one selected at the current iteration. If there exists a subset of channels $\mathcal{L}_{i}^{*} \subseteq \mathcal{L}_{i}$ such that each channel of which preserves (if assigned alone to MR $i$ ) the connectivity in $\mathbf{C}_{(u)}$ and $\mathbf{C}_{(d)}$ (lines 6-11), then MR $i$ must be assigned a channel from $\mathcal{L}_{i}^{*}$. From $\mathcal{L}_{i}^{*}$, channels that were not assigned to adjacent cells are preferred over other channels. The channel $\hat{k}$ with the maximum weight, i.e., $\mathbf{W}[i, \hat{k}]$, is selected, and ties are broken based on the number of MRs that can access MR $i$ on $\hat{k}$.

If subset $\mathcal{L}_{i}^{*}$ is empty (which means that there is no channel that if assigned to MR $i$, the connectivity will be preserved), then the channel that allows the maximum number of neighboring MRs to access MR $i$ is selected (lines 12-13).

After allocating a channel to MR $i$, the connectivity vectors $\mathbf{C}_{(u)}$ and $\mathbf{C}_{(d)}$ are updated. Also, all the MCs that belong to $\mathcal{A}_{i}$ and cannot access MR $i$ on the selected channel $\hat{k}$ must be removed. Moreover, all MCs that have their parent MRs disconnected from the backbone network (either in the upstream or the downstream direction) must be removed.

\section{B. Phase 2: Finding the maximum number of reliable uplinks}

Before we move into the second phase of our solution strategy, we propose a power control algorithm (PCA). The PCA algorithm takes as an input two sets of links: a set of uplinks $\mathcal{Q}_{u}(k)$ and a set of downlinks $\mathcal{Q}_{d}(k)$, as well as the channel $k$ on which those links operate. If there exists a power allocation for all links' transmitters such that the SINR at all links' receivers is at least $\gamma$, then the algorithm returns 1 , otherwise it returns 0 . To test the existence of a feasible power allocation (one that achieves the reliability of all links), we propose a simple linear programming (LP) formulation that aims at finding any feasible solution, i.e., no optimization objective. The LP is outlined in Algorithm 2. The first and the second constraints correspond to the interference caused

\footnotetext{
${ }^{5}$ We assume a single gateway in the system. However, the algorithm can be extended to the case of multiple gateways by adding a hypothetical one that has all channels available and is connected to all other gateways.
} 


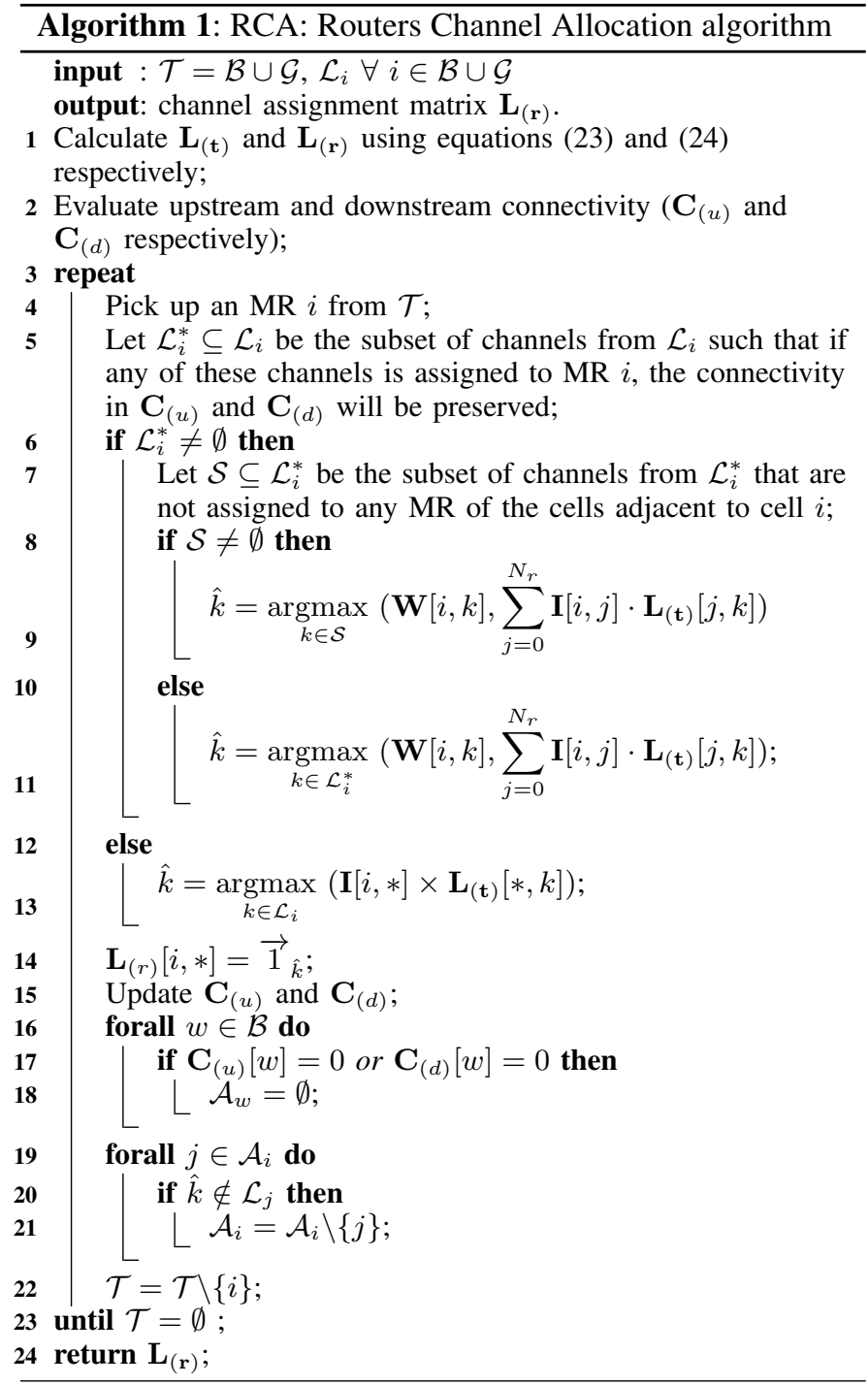

by active cells at the receivers in other active cells (similar to the inter-cell constraints (19) and (20) in Section IV. An active cell is a cell that has at least one link in $\mathcal{Q}_{u}(k) \cup \mathcal{Q}_{d}(k)$. The third and the fourth constraints, on the other hand, correspond to the reliability requirement of the uplinks and downlinks respectively. $r(e)$ and $t(e)$ denote the receiver node and the transmitter node of link $e$ respectively. Now, we can explain our solution for the second phase, i.e., maximizing the number of reliable uplinks. This phase is outlined in lines [6-13] in Algorithm 3. The output of the first phase is the allocation of exactly one channel $k \in \mathcal{L}_{i}$ for each MR $i \in \mathcal{B} \cup \mathcal{G}$. The idea is to go over the channels in $\mathcal{L}$ one by one. For each channel $k$, we find the set of potential uplinks on channel $k$, denoted as $\mathcal{Q}_{u}(k)$, as shown in line 7. If $\mathcal{Q}_{u}(k)$ is not empty, then for each uplink $e$, we find the maximum channel gain, $\lambda_{e}$, between $t(e)$ and all the receiving MRs in $\mathcal{Q}_{u}(k)$ except $r(e)$ as follows:

$\lambda_{e}=\max _{i: \exists(j, i) \in \mathcal{Q}_{u}(k), i \neq r(e)} \Psi_{t(e), i}^{k}$

Then, we process the uplinks in $\mathcal{Q}_{u}(k)$ in ascending order of their $\lambda_{e}$ values. For each uplink, we use the PCA algorithm to find out whether it can be supported, i.e., reliably served, without affecting, i.e., breaking the reliability of, already

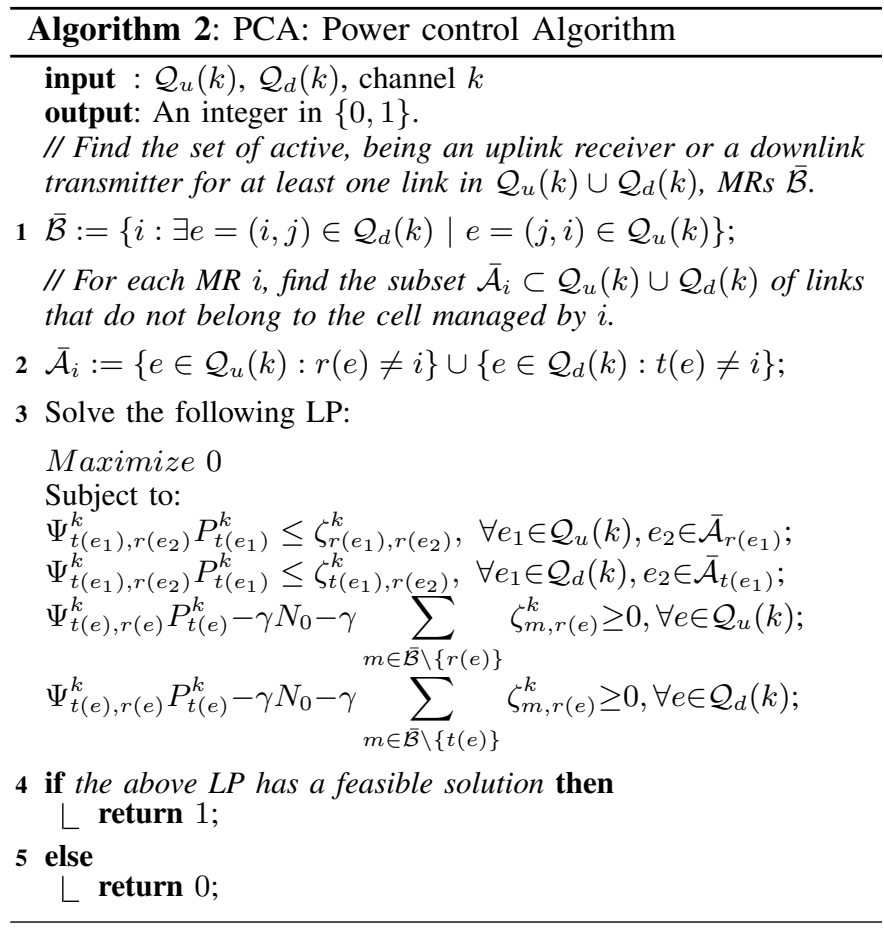

reliably served uplinks. If so, the uplink is added to the set of reliable uplinks $\mathcal{Q}_{u}^{r}(k)$, otherwise it will not be added.

\section{Phase 3: Channel allocation to MCs}

The last phase is channel allocation to MCs, i.e., downlinks. First of all, the MCs to be considered in the phase are only the ones that have reliable uplinks with their parent MRs after the second phase. Therefore, in lines [15-16] of Algorithm 3, we set $A_{i} \forall i \in \mathcal{B} \cup \mathcal{G}$ to those MCs that have reliable uplinks with MR $i$. Similar to what we did with uplinks, we need to process potential downlinks in ascending order of their maximum channel gains. However, the case now is different. Each MC may have several channels available, i.e., $\mathcal{L}_{j}>1$ for $j \in \mathcal{A}$. This provides us with multiple choices for each downlink, in contrary to the uplink case where each uplink has only one choice, i.e., the channel assigned to the MR of that uplink. Therefore, for each MC $j$, we will find $\left|\mathcal{L}_{j}\right|$ maximum channel gains each on one of the channels in $\mathcal{L}_{j}$. Let $\mathcal{P}$ be the set of all possible $(M C$, channel) pairs defined as follows:

$\mathcal{P}=\left\{(i, k): i \in \bigcup_{j \in \mathcal{B} \cup \mathcal{G}} \mathcal{A}_{j}, k \in \mathcal{L}_{i}\right\}$

Recall that this set is evaluated after removing MCs that cannot be served on the uplink. Therefore, all MCs represented by at least one pair in $\mathcal{P}$ have passed the second phase, i.e., can be served reliably on the uplink. Let $p(i)$ denote the parent MR of MC $i$. Then for each pair $(i, k) \in \mathcal{P}$, the maximum channel gain $\lambda_{(i, k)}$ is calculated as follows:

$$
\begin{aligned}
& \lambda_{(i, k)}= \\
& \left.\max _{j:(j, k) \in \mathcal{P}, p(i) \neq p(j)} \Psi_{i j}^{k}, \max _{j \in \mathcal{B} \cup \mathcal{G} \backslash\{p(i)\}: \exists(m, j) \in \mathcal{Q}_{u}^{r}(k)} \Psi_{i j}^{k}\right\}
\end{aligned}
$$

The above equation finds the maximum channel gain $\lambda_{(i, k)}$ on channel $k$ between MC $i$ and any other MC that has channel $k$ available or a MR that was assigned channel $k$ in the first 
phase. Then, we process the pairs in $\mathcal{P}$ in ascending order of their maximum channel gains. For each pair $(i, k)$, we add the downlink $(p(i), i)$ to the current set of reliable downlinks on channel $k, \mathcal{Q}_{d}^{r}(k)$ (initially empty), and the uplink $(i, p(i))$ to the current set of reliable uplinks on channel $k^{\prime}, \mathcal{Q}_{u}^{r}\left(k^{\prime}\right)$ (which is initially empty) where $k^{\prime}$ is the channel assigned to $p(i)$, i.e, $\mathbf{L}_{(r)}\left[i, k^{\prime}\right]=1$. Using the PCA algorithm, if both the uplink and the downlink can be served reliably without breaking the reliability of any link in $\mathcal{Q}_{d}^{r}(k)$ and $\mathcal{Q}_{u}^{r}\left(k^{\prime}\right)$, then this MC is added to the set of reliable MCs $\mathcal{A}^{r}$ and the downlink and the uplink are admitted to the set $\mathcal{Q}_{d}^{r}(k)$ and $\mathcal{Q}_{u}^{r}\left(k^{\prime}\right)$ respectively. Otherwise, the two links will be removed from $\mathcal{Q}_{d}^{r}(k)$ and $\mathcal{Q}_{u}^{r}\left(k^{\prime}\right)$ and the $\mathrm{MC}$ will not be added to $\mathcal{A}^{r}$. Once an MC is added to $\mathcal{A}^{r}$ by one of its pairs, other pairs of this MC in $\mathcal{P}$ will be ignored. This process is presented in lines [21-34] in Algorithm 3. Finally, to find a final power allocation for MRs and MCs, we run the PCA algorithm once for each channel over the set of reliable uplinks and downlinks on that channel.

The algorithm that combines all the three phases together is presented in Algorithm 3, which we call the heuristic receiverbased channel allocation (HRBA) algorithm.

\section{PRACTICAL IsSUES}

\section{A. Medium access control}

There is a need to devise a MAC mechanism that can work with the proposed RBA strategy. Designing such a mechanism is challenged by the three major issues which we have discussed earlier in Section III-A. In this section, we discuss some of the possible medium access solutions that can be adopted under the proposed RBA strategy.

1) Synchronized Hybrid MAC: A hybrid TDMACSMA/CA mechanism can be used to overcome all of the three aforementioned challenges. The basic idea is to assign each SU a time slot during which it acts as a receiver only. At the beginning of the slot assigned to a particular SU, say $i$, all other SUs that want to communicate with $i$ must tune to the channel assigned to $i$. Then, they contend to gain access to that channel using the traditional CSMA/CA with RTS/CTS handshake. Although such an approach overcomes all of the aforementioned challenges, it requires centralized scheduling, which makes it inflexible especially with dynamically changing traffic rates.

2) Synchronized MAC Protocol For Multi-Hop Cognitive Radio Networks (Sync-MAC): Reference [47] proposed a synchronized MAC protocol for multihop cognitive radio networks that does not require a common control channel. This protocol assumes that nodes are equipped with two radio transceivers. One of the two radios is dedicated to listening to control signals, while the other is used for transmitting and receiving data. Each of the channels is assigned dedicated time slot in a periodic fashion. The slot is long enough to exchange control signals to coordinate the communication between a pair of nodes (like RTS-CTS). When node $A$ wants to transmit to node $B$, it chooses a slot on a common channel between the two nodes, starts a backoff counter, and when the backoff expires it sends an RTS if the channel is idle. $B$ replies with the CTS, and then communication starts. Nodes that have heard either the RTS or the CTS will realize that both nodes will not

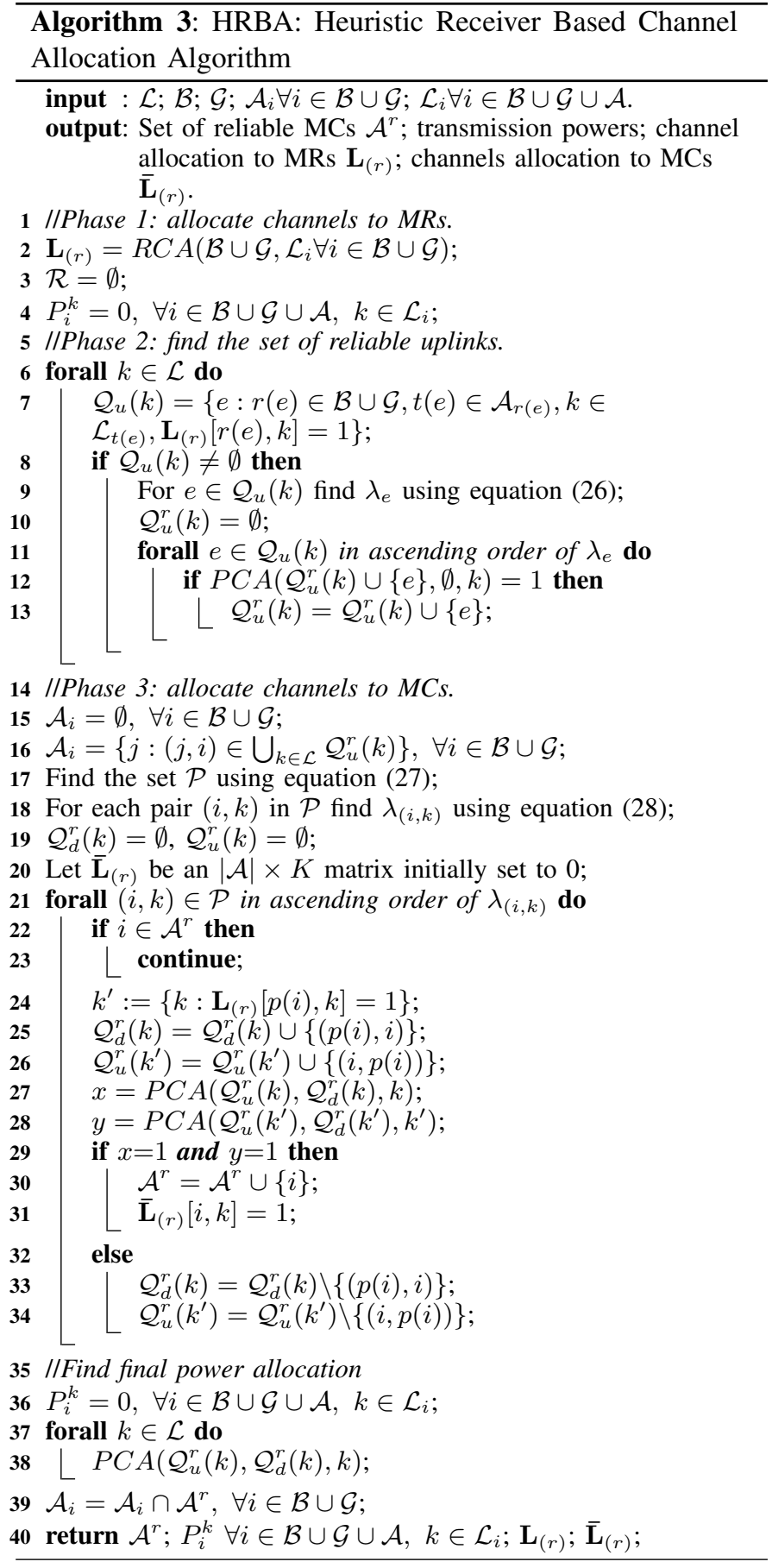

be available for the NAV value (Network Allocation Vector). Note that no node will miss any control packets transmitted over a particular channel at any time, even if it is currently transmitting on another channel, because it has a dedicated radio for that purpose (i.e., listening to control messages). This protocol can be used for medium access control under the RBA allocation strategy. The only difference here is that each node can receive on one channel only. According to [47], this MAC protocol outperforms CCC-based MACs in terms of both throughput and connectivity.

The problem in using this MAC protocol for the specific network studied in this paper is that it does not guarantee that multihop transmissions (between MRs) will not cause interference to single-hop transmissions (those between an 
MR and the MCs it serves). Our MILP formulations assumed disjointness between multihop and single hop transmissions. Another drawback is the two radios per node requirement. Therefore, we propose a modified version of Sync-MAC.

3) Synchronized MAC for RBA: We now present a modified version of the synchronous MAC protocol discussed in the previous subsection which does not require two radio transceivers at the MCs and at the same time protects upstream/downstream links within each cell from interference caused by multihop communication. Initially, MRs use the synchronized MAC in its original form proposed in [47], with one difference which is that the time is divided intro frames as shown in Figure 4. The purpose of dividing the time into equal frames is to control the interference between multihop and single hop links. An MR operates in two modes, the intercell (multihop) mode, and the intra-cell (single hop mode). In the former, it exchanges data with its neighboring MRs using the Sync-MAC discussed earlier. When an MR wants to exchange data with MCs, it switches into the intra-cell mode. This switching can be done only at the beginning of a frame to keep all intra-cell transmissions across cells aligned, and so easy to protect. Before switching into the intra-cell mode, an MR must inform the neighboring MRs about its intention to schedule a periodic intra-cell communication subframe to disseminate/collect data to/from MCs. The bandwidth allocated to intra-cell communication (i.e., the percentage of time allocated to intra-cell communication) depends on the number of MCs associated with that cell and can be regulated at the network level. The MR can later change the length or frequency of this intra-cell communication period depending on the activity of the MCs in the intended cell. Adjacent MRs will avoid doing inter-cell communication on a channel, say $k$, during the intra-cell communication period of an adjacent cell if the latter has an uplink/downlink that used channel $k$.

We now describe the intra-cell communication and how it works. To initiate the intra-cell period for the first time, the MR notifies the MCs individually on their channels with an INITIATE control packet. This packet synchronizes the MCs with their parent MR, informs them about when the period will start and about its length, and finally the order of their assigned control mini slots shown in Figure 4. Each MC is assigned one mini slot during which it informs the MR about the number of packets it has ready for transmission, including control packets. The MR then calculates a schedule for the current period, and polls the MCs individually. The polling starts with a poll packet transmitted by the MR on the channel assigned to the MC. The MC then replies with its data packet to the MR. Finally the MR sends back the ACK, and then polls the next MC. This operation is summarized in Figure 5.

The performance of this MAC protocol depends on how the parameters are selected. To give an example that illustrates this dependency, we simulated a network of one MR and ten MCs. The simulator parameters are summarized in Table II.

We simulated unidirectional traffic (from MCs to MR) under different arrival rates and measured the aggregate throughput as well as the average latency. The frame length is always fixed, while we vary the length of the intra-cell sub-frame. We also vary the packet mean arrival rate, and consider

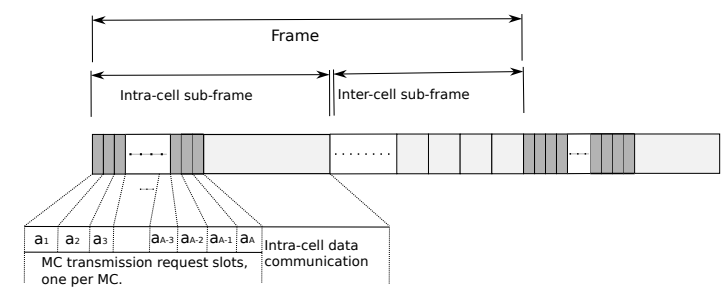

Fig. 4. The format of the modefied Sync-MAC frame

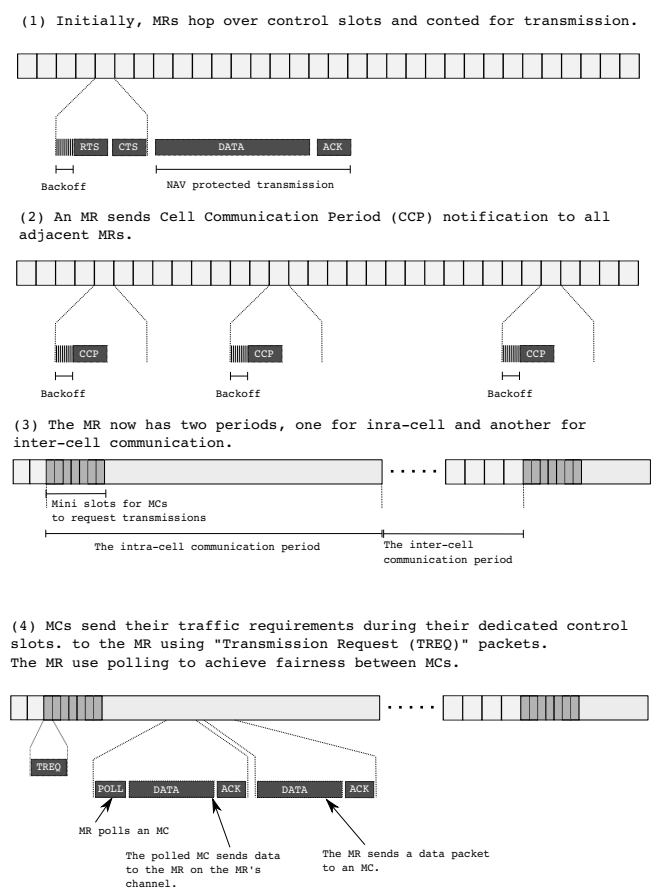

Fig. 5. The operation of the MAC-RBA mechanism.

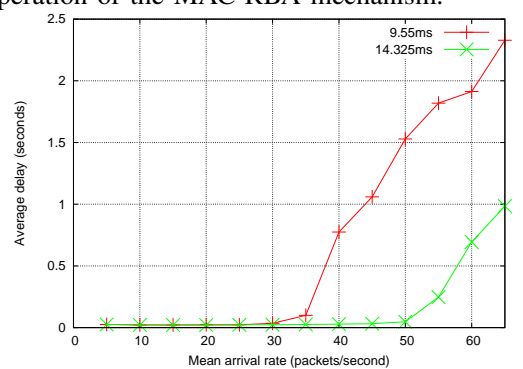

Fig. 9. The average delay under different lengths of the intra-cell communication period, and exponentially distributed packet inter-arrival time.

both cases of fixed and exponentially distributed packet interarrival time. The aggregate throughput under the fixed and exponentially distributed packet inter-arrival time are shows in figures 6 and 8 respectively, while the average delay results are shown in Figures 7 and 9 respectively. As the figure shows, the aggregate cell throughput depends on the amount of time allocated to that cell for intra-cell communication. Increasing the percentage of the frame time dedicated to intracell communication from $34.67 \%$ to $52 \%$ resulted in $\approx 50 \%$ increase in throughput and $\approx 200 \%$ decrease in latency.

\section{B. Dissemination of control data}

To facilitate the dissemination process, several solutions can be proposed. One possible solution is to rely on the SyncMAC in its original form. Before any channels are allocated, the nodes can use the original Sync-MAC to disseminate the 


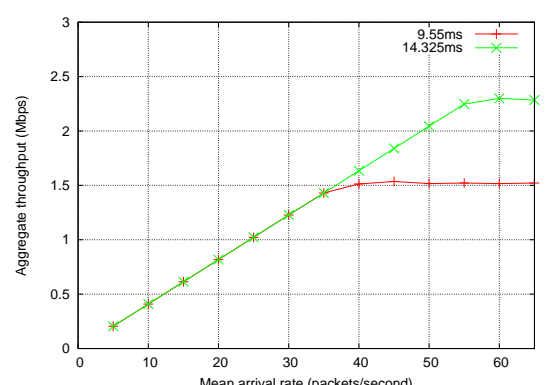

Fig. 6. The aggregate throughput under different intra-cell communication periods, and fixed packet inter-arrival time.

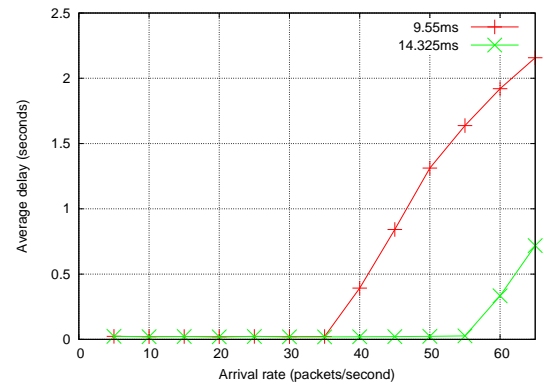

Fig. 7. The average delay under different intracell communication periods, and fixed packet inter-arrival time.

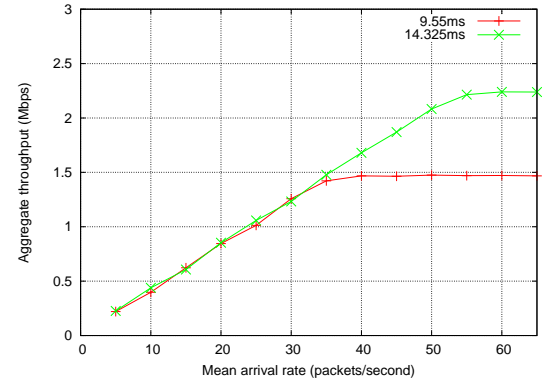

Fig. 8. The aggregate throughput under different intra-cell communication periods, and exponentially distributed packet inter-arrival time. data from/to the central router (the gateway MR) where the channel allocation is calculated. Once each node is allocated a channel, the network switches to our modified version of the Sync-MAC which we proposed in the previous subsection. This switching shall be initiated by the gateway MR as well. On the other hand, this dissemination should not have a significant impact on the bandwidth because of the assumption that channel status is quasi-static, and hence new schedules will not be disseminated very often as well.

\section{Fairness between SUs}

Depending on how the channels are used (when and by which PUs), the service (channel) availability may be different at different SUs, resulting in an unfair service.

Taking fairness into account while assigning channels requires a model to predict the channel occupation pattern of PUs. Predicting when PUs will occupy which channels is not an easy task, especially when coupled with the RBA optimization problem. Because we assume that channel status is quasi static, the algorithm will be rerun in such cases, which will make the fairness issue less of a problem.

\section{The effect of switching latency}

MRs may suffer from performance issues due to the switching overhead if they have to relay multiple flows on different channels to different neighboring MRs. This issue can be solved as follows: Add one more constraint to the MILP formulation such that if an MR receives on channel $k$, it can transmit on channel $m$ only if $|m-k| \leq \alpha$, where $\alpha$ is the maximum separation between channels to prevent excessive switching latency. Changing our heuristic algorithms to work this way is straightforward. We only need to change the routers channel allocation algorithm, viz., Algorithm 1. After line 14 in Algorithm 1, we need to add the following action:

$L_{(t)}[i, k]=0 \forall k \in \mathcal{L}_{i}:|k-\hat{k}| \leq \alpha$.

which restricts the set of channels that MR $i$ can transmit on to those which are not far away from the channel selected for reception (i.e., $\hat{k}$ ). Such an approach will limit the coverage of the network as well as the connectivity with the gateway. Therefore, for extended coverage and connectivity, we have to pay the price in terms of switching latency.

\section{Performance of THE HRBA Algorithm}

In this section, we compare the optimal performance of all the three allocation strategies proposed in Section IV in terms of the number of MCs served, and evaluate the performance of the proposed HRBA algorithm. To vary the channel availability distribution at different SUs, we are varying the number and locations of PUs. The network is deployed in an $A \times A$ square area. The area is divided into $N_{r}$ cells, such that $N_{r}=|\mathcal{B}|+|\mathcal{G}|$. We obtained the optimal solutions for $N_{r}=4$, and $9 \mathrm{MRs}$ and $100 \mathrm{MCs}$. In all scenarios, we assume the existence of a single gateway MR located at the right-bottom cell. The number of PUs is varied to achieve different channel availability distributions. Each PU is randomly assigned one of the $K$ orthogonal channels available in the system. A SU (an $\mathrm{MC}$ or an MR) cannot use channel $k$ if the user is less than $R_{p}$ apart from a PU that is assigned channel $k . R_{p}$ is set equal to the cell radius, i.e., $R_{p}=\frac{A}{2 \sqrt{N_{r}}}$. The maximum transmission power of an MR is calculated as $\left(\frac{2.5 A}{2 \sqrt{N_{r}}}\right)^{\alpha} N_{0} \gamma$ and of an MC as $\left(\frac{A}{\sqrt{N_{r}}}\right)^{\alpha} N_{0} \gamma$, where $\gamma=15 d B$ and $N_{0}=10^{-11}$ Watt. These values will guarantee that each MR will be able to reach the four adjacent (up, down, left, and right) MRs, and that each MC will be able to reach its parent MR. For all the experiments in this section, the path-loss exponent $\alpha=3.76$. For each topology, we randomize the locations of PUs and MCs as well as the channels allocated to PUs to generate different channel availabilities at MCs and MRs.

\section{A. Without a preassumed CCC}

We first study the optimal performance of the three allocation strategies without presuming the existence of a CCC in the network. Figures 10 and 12 show the optimal performance of the three strategies for the case of $4 \mathrm{MRs}$ and $9 \mathrm{MRs}$ respectively. The number of active PUs is varied from 15 to 40 for the case of $4 \mathrm{MRs}$, and from 30 to 55 for the case of 9 MRs. Each point on the curves is the average of 100 randomly generated topologies with a $95 \%$ confidence. As the figures imply, RBA outperforms the other two approaches. Notice that the difference in performance between RBA and ATA is higher for fewer PUs. For instance, the number of served MCs using RBA, in Figure 12, is on average 1.5 times that using ATA for 30 PUs, however, this number jumps to 3.5 for the case of 55 PUs. The TBA approach, on the other hand, is always outperformed by ATA. This is expected because they both require a $\mathrm{CCC}$, but ATA can use all the channels for transmission while TBA is restricted to one channel.

\section{B. With a preassumed $C C C$}

In this subsection, we evaluate the performance of the three allocation strategies with the presumption that a CCC exists. 
TABLE II

PARAMETERS TO EVALUATE THE "synchronized MAC for RBA"

\begin{tabular}{|l|l||l|l|}
\hline Parameter & Value & Parameter & Value \\
\hline$\gamma$ & $15 d B$ & $N_{O}$ & $10^{-11}$ Watt \\
$\alpha$ & 3.76 & Frame length & $27.55 \mathrm{~ms}$ \\
Data packet size & $512 B$ & Data+Poll+Ack time & $0.9 \mathrm{~ms}$ \\
Mini-slot length & $50 \mu \mathrm{s}$ & Number of MCs & 10 \\
\hline
\end{tabular}

We add one more channel and make it available to all nodes, i.e., no PU can use this extra channel. Figures 11 and 13 show the optimal performance of the three strategies for the case of 4 MRs and 9 MRs respectively. The number of PUs is varied from 15 to 40 for the case of 4 MRs, and from 30 to 55 for the case of 9 MRs. Again, each point on the curve is an average of 100 randomly generated topologies with a $95 \%$ confidence. As the figures indicate, the RBA still outperforms the other two strategies even though a CCC is preassumed. However, the difference between RBA and the other approaches is less in this case than the case when no $\mathrm{CCC}$ is preassumed. The figures also show that for fewer PUs (which means high channel availability), the performance of the RBA strategy is very close to that of the ATA strategy.

\section{Performance of the HRBA algorithm}

In this subsection, we compare the performance of the HRBA algorithm to the optimal performance obtained using the MILP formulation in Section IV. In Figure 14, we show the number of served MCs (the average over a 100 randomly generated topologies with a $95 \%$ confidence) obtained using the HRBA algorithm and the MILP formulation for $|\mathcal{B}|=8$, $|\mathcal{G}|=1,|\mathcal{A}|=100$ and $K=6$. As the figure shows, the performance of the HRBA algorithm is close to the optimal solution, within with $\approx 14.7 \%$ of the optimal (on average). Figure 15 shows the same results for $|\mathcal{B}|=15,|\mathcal{G}|=1$, $|\mathcal{A}|=100$ and $K=6$. Again, the HRBA algorithm is, on average, within $\approx 12 \%$ of the optimal solution.

\section{CONCLUSIONS}

We have studied, in this work, the channel allocation problem in wireless cognitive mesh networks. By controlling the tunability of the transmission and reception parts of the cognitive radio, four different modes of operation were defined for cognitive transceivers. Three channel allocation strategies based on the aforementioned modes were defined, namely receiver-based allocation $R B A$, transmitter-based allocation TBA, and all-tunable allocation ATA. MILP formulations were proposed RBA and ATA strategies with the objective of maximizing the number of served MCs with a reliability guarantees on the uplink and downlink for each MC. However, the MILP for the TBA case was omitted for lack of space. Results show that the proposed RBA strategy outperforms the TBA and the ATA strategies even when a CCC is preassumed.

We also proposed a heuristic solution for the RBA problem. Results show that the performance of the proposed algorithm is, on average, within $28 \%$ of the optimal solution.

\section{REFERENCES}

[1] Ian F. Akyildiz et al. Next generation/dynamic spectrum access/cognitive radio wireless networks: a survey. Comput. Netw., 50(13):2127-2159, 2006.
TABLE III

SIMULATION PARAMETERS TO EVALUATE THE HRBA ALGORITHM

\begin{tabular}{|l|l||l|l|}
\hline Parameter & Value & Parameter & Value \\
\hline$\gamma$ & $15 d B$ & $N_{r}$ & 4 \\
$N_{0}$ & $10^{-11}$ Watt & $P_{r}^{\max }$ & $\left(\frac{2.5 A}{2 \sqrt{N_{r}}}\right)^{\alpha} N_{0} \gamma$ \\
$\alpha$ & 3.76 & $P_{c}^{\max }$ & $\left(\frac{A}{\sqrt{N_{r}}}\right)^{\alpha} N_{0} \gamma$ \\
\hline
\end{tabular}

[2] Joseph Mitola. An Integrated Agent Architecture for Software Defined Radio. PhD thesis, Royal Institute of Technology (KTH), Stockholm, Sweden, May 2000

[3] J. Zhao, H. Zheng, and G.-H. Yang. Distributed coordination in dynamic spectrum allocation networks. First IEEE International Symposium on New Frontiers in Dynamic Spectrum Access Networks (DySPAN), 2005.

[4] T. Chen et al. Topology management in cogmesh: A cluster-based cognitive radio mesh network. IEEE International Conference on Communications (ICC), pages 6516-6521, June 2007.

[5] Y. R. Kondareddy and P. Agrawal. Synchronized mac protocol for multi-hop cognitive radio networks. IEEE International Conference on Communications (ICC'08), pages 3198-3202, May 2008.

[6] Y. R. Kondareddy and P. Agrawal. Selective broadcasting in multi-hop cognitive radio networks. IEEE Sarnoff Symposium, pages 1-5, 2008.

[7] K.R. Chowdhury and I.F. Akyildiz. Cognitive wireless mesh networks with dynamic spectrum access. IEEE Journal on Selected Areas in Communications, 26(1):168-181, Jan. 2008.

[8] Zhou Yuan, Ju Bin Song, and Zhu Han. Interference minimization routing and scheduling in cognitive radio wireless mesh networks. In Proceedings of WCNC, 2010.

[9] C.-C. Shen C. Xin, L. Ma. A path-centric channel assignment framework for cognitive radio wireless networks. In Mobile Networks and Applications, 2008.

[10] Q. Wang and H. Zheng. Route and spectrum selection in dynamic spectrum networks. In 3rd IEEE Consumer Communications and Networking Conference (CCNC), pages 625-629, 2006.

[11] X. Zhou, L. Lin, J. Wang, and X. Zhang. Cross-layer routing design in cognitive radio networks by colored multigraph model. Wireless Personal Communications, 49(1):pages 123-131, 2009.

[12] Y. Xi and E. M. Yeh. Distributed algorithms for spectrum allocation, power control, routing, and congestion control in wireless networks. In ACM MobiHoc, pages 180-189, 2007.

[13] Y. Shi and Y. T. Hou. A distributed optimization algorithm for multihop cognitive radio networks. In IEEE INFOCOM, page 12921300, 2008.

[14] R. Hincapie et al. Qos routing in wireless mesh networks with cognitive radios. In IEEE GLOBECOM, pages 1-5, 2008.

[15] Amr A. El-Sherif and Amr Mohamed. Delay minimization through joint routing and resource allocation in cognitive radio-based mesh networks. In GlobeCom 2012, Ad Hoc and Sensor Networking Symposium, pages 403-409, 2012.

[16] Matteo Cesana et al. Routing in cognitive radio networks: Challenges and solutions. Ad Hoc Networks, 2010.

[17] Brandon F. Lo. A survey of common control channel design in cognitive radio networks. Journal Physical Communication, 4, Issue 1:26-39, March 2011.

[18] N. Baldo, A. Asterjadhi, and M. Zorzi. Dynamic spectrum access using a network coded cognitive control channel. IEEE Transactions on Wireless Communications, 9(8):2575-2587, 2010.

[19] Claudia Cormio and Kaushik R. Chowdhury. Common control channel design for cognitive radio wireless ad hoc networks using adaptive frequency hopping. Journal Ad Hoc Networks, 8(4):430-438, June 2010.

[20] Tao Chen et al. Cogmesh: A cluster-based cognitive radio network. In 2nd IEEE International Symposium on New Frontiers in Dynamic Spectrum Access Networks (DySPAN), pages 168-178, 2007.

[21] C. Doerr, D. Grunwald, and D.C. Sicker. Dynamic control channel management in presence of spectrum heterogeneity. In IEEE Military Communications Conference (MILCOM), pages 1-8, 2008.

[22] B. Hamdaoui and K.G. Shin. Os-mac: An efficient mac protocol for spectrum-agile wireless networks. IEEE Transactions on Mobile Computing, 7(8):915-930, 2008.

[23] A. Motamedi and A. Bahai. Mac protocol design for spectrumagile wireless networks: Stochastic control approach. In 2nd IEEE International Symposium on New Frontiers in Dynamic Spectrum Access Networks, 2007.

[24] P. Kyasanur, Jungmin So, C. Chereddi, and N.H Vaidya. Multichannel mesh networks: challenges and protocols. In IEEE Wireless Communications, volume 13, pages 30-36, April 2006.

[25] P. Kyasanur et al. Net-x: System extensions for supporting multiple channels, multiple interfaces, and other interface capabilities. In Technical Report, University of Illinois at Urbana-Champaign, Aug. 2006. 


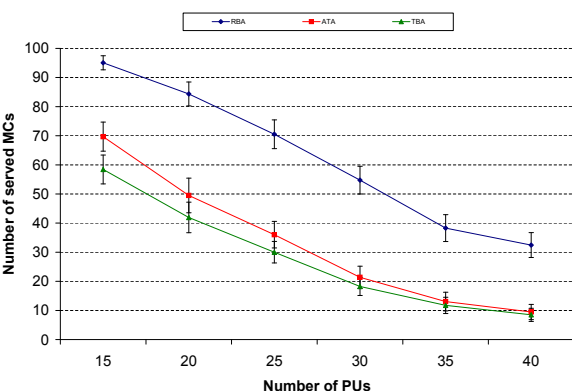

Fig. 10. The performance of the three allocation strategies without a preassumed CCC. $|\mathcal{B}|=3$, $|\mathcal{G}|=1,|\mathcal{A}|=100$, and $K=6$.

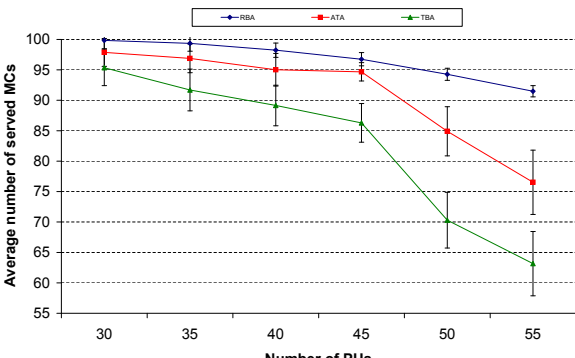

Fig. 13. The performance of the three allocation strategies with a preassumed CCC. $|\mathcal{B}|=8,|\mathcal{G}|=$ $1,|\mathcal{A}|=100$, and $K=7$.
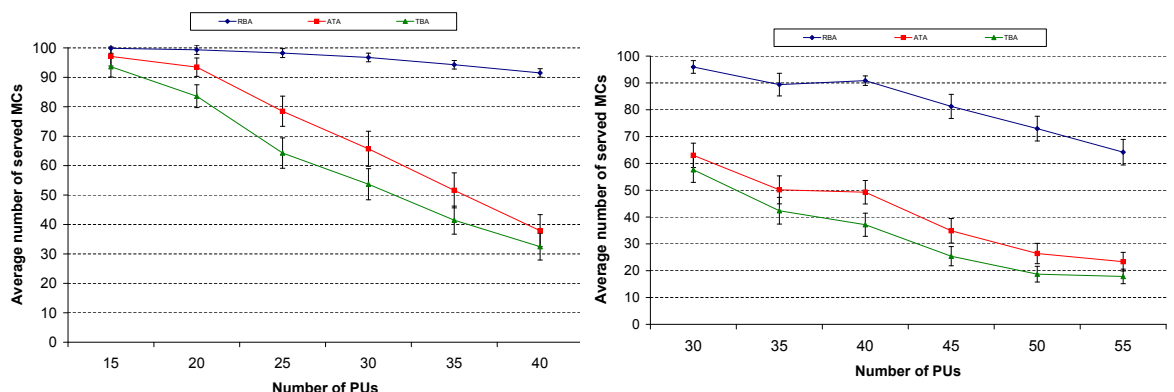

Fig. 12. The performance of the three allocation Fig. 11. The performance of the three allocation strategies without a preassumed CCC. $|\mathcal{B}|=8$, strategies with a preassumed CCC. $|\mathcal{B}|=3,|\mathcal{G}|=|\mathcal{G}|=1,|\mathcal{A}|=100$, and $K=6$.
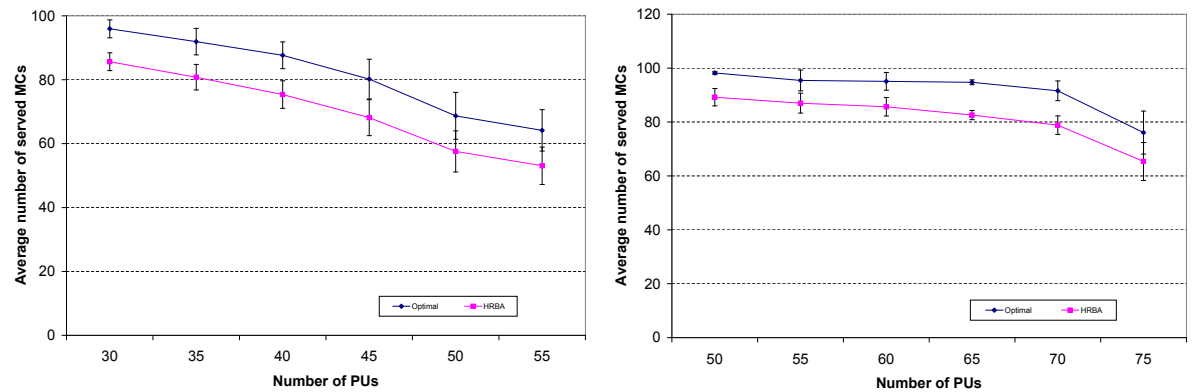

Fig. 14. The performance of the HRBA algorithm Fig. 15. The performance of the HRBA algorithm compared to the optimal solution. $|\mathcal{B}|=8,|\mathcal{G}|=$ compared to the optimal solution. $|\mathcal{B}|=15,|\mathcal{G}|=$ $1,|\mathcal{A}|=100$, and $K=6$. $1,|\mathcal{A}|=100$, and $K=6$.
[26] H. Skalli et al. Channel assignment strategies for multiradio wireless mesh networks: issues and solutions. In IEEE Communications, 2007. vol. 45, pp. 86-95.

[27] P. Kyasanur and N.F. Vaidya. Routing and interface assignment in multi-channel multi-interface wireless networks. In IEEE Wireless Communications and Networking Conference (WCNC), 2005.

[28] Pradeep Kyasanur. Routing and link-layer protocols for multi-channel multi-interface ad hoc wireless networks. Sigmobile Mobile Computing and Communications Review, 10:31-43, 2006.

[29] P.H. Pathak and R. Dutta. A survey of network design problems and joint design approaches in wireless mesh networks. IEEE Communications Surveys Tutorials, 13(3):396 -428, quarter 2011.

[30] A. Adya et al. A multi-radio unification protocol for ieee 802.11 wireless networks. In Proceedings. First International Conference on Broadband Networks, pages 344-354, October 2004.

[31] M. Marina and S. Das. A topology control approach for utilizing multiple channels in multi-radio wireless mesh networks. In Computer Networks, volume 54, pages 241-256, 2010.

[32] A. Subramanian, H. Gupta, , and S. Das. Minimum interference channel assignment in multi-radio wireless mesh networks. In 4th IEEE Communications Society Conference on Sensor, Mesh and Ad Hoc Communications and Networks (SECON), pages 481-490, June 2007.

[33] A. Sen et al. An interferenceaware channel assignment scheme for wireless mesh networks. In IEEE International Conference on Communications (ICC), pages 3471-3476, June 2007.

[34] A. H. M. Rad and V. W. S. Wong. Wsn16-4: Logical topology design and interface assignment for multi-channel wireless mesh networks. In IEEE Global Telecommunications Conference (GLOBECOM), 2006.

[35] Mansoor Alicherry et al. Joint channel assignment and routing for throughput optimization in multiradio wireless mesh networks. In IEEE Journal on Selected Areas in Comm., 2006. vol. 24, pp. 1960-1971.

[36] Lin Gao, Xinbing Wang, and Youyun Xu. Multiradio channel allocation in multihop wireless networks. IEEE Transactions on Mobile Computing, 8(11):1454 -1468, November 2009 .

[37] Jun Luo et al. Engineering wireless mesh networks: Joint scheduling, routing, power control, and rate adaptation. IEEE/ACM Transactions on Networking, 18(5):1387-1400, October 2010.

[38] A. T. Hoang and Y.-C. Liang. Downlink channel assignment and power control for cognitive radio networks. In IEEE transactions on wireless communications, volume 7, August 2008.

[39] M. Sharma, A. Sahoo, and K.D. Nayak. Channel selection under interference temperature model in multi-hop cognitive mesh networks. In DySPAN, pages 133-136, April 2007.

[40] M. Haidar, M. Msakni, and Z. Dziong. Power management and bandwidth allocation in a cognitive wireless mesh network. In Seventh Annual Communication Networks and Services Research Conference (CNSR), pages 391-396, 2009.

[41] Zhijin Zhao, Zhen Peng, Zhidong Zhao, and Shilian Zheng. Joint power control and spectrum allocation for cognitive radio with qos constraint. Communication and Network, 2(1):38-43, 2010.

[42] V. Fodor, I. Glaropoulos, and L. Pescosolido. Detecting low-power primary signals via distributed sensing to support opportunistic spectrum access. IEEE International Conference on Communications (ICC), 2009.

[43] B. Mercier, V. Fodor, et al. Sensor networks for cognitive radio: Theory and system design. ICT Mobile Summit, June 2008.

[44] R. Thobaben and E. G. Larsson. Sensor-network-aided cognitive radio: On the optimal receiver for estimate-and-forward protocols applied to the relay channel. In The Forty-First Asilomar Conference on Signals, Systems and Computers (ACSSC), 2007.

[45] H. Gossain et al. The deafness problems and solutions in wireless ad hoc networks using directional antennas. IEEE Global Telecommunications Conference Workshops, pages 108-113, 2004.

[46] Hisham M. Almasaeid. Spectrum Allocation Algorithms for Cognitive Radio Mesh Networks. PhD thesis, Iowa State University, 2011.

[47] Y.R. Kondareddy and P. Agrawal. Synchronized mac protocol for multihop cognitive radio networks. In IEEE International Conference on Communications (ICC), pages 3198 -3202, May 2008.

[48] P. Hansen and B. Jaumard. Algorithms for the maximum satisfiability problem. Computing, 44:279 -303, 1990.

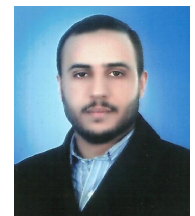

Hisham M. Almasaeid is an assistant professor of Computer Engineering at Yarmouk University, Jordan. He received his $\mathrm{PhD}$ from Iowa State University in Fall 2011. His research interests include Cognitive Radio Networks, Wireless Sensor Networks, and Mobile Ad Hoc Networks.

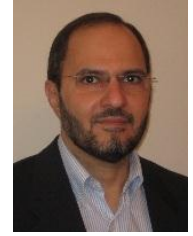

Ahmed E. Kamal (S'82-M'87-SM'91-F'12) is a professor of Electrical and Computer Engineering at Iowa State University. His research interests include Cognitive Radio Networks, Wireless Sensor Networks and Optical Networks. 


\section{SUPPLEMENTARY DOCUMENT: THE COMPLEXITY OF THE UDCP PROBLEM}

To prove the NP-hardness of the UDCP problem, we first prove the NP-completeness of a decision version of UDCP termed D-UDCP. The NP-completeness of the D-UDCP is proven by a reduction from the Maximum Satisfiability (MAX-SAT) problem. Before proceeding with the proof, we give the definitions of both the MAX-SAT and the D-UDCP problems.

Definition 1 [MAX-SAT] Given a set of boolean variables $\mathcal{X}=\left\{x_{1}, \cdots, x_{N}\right\}$, each of which appears in at least one clause from the set of clauses $\mathcal{C}=\left\{C_{1}, \cdots, C_{M}\right\}$, where a clause is the OR operation of a number of variables each of which appears in either a negative of a positive form. For an integer $k \leq M$, is there a boolean assignment for the $N$ variables that satisfies at least $k$ clauses (i.e., make them evaluate to TRUE)? Let us denote this problem as MAX-SAT $\langle\mathcal{X}, \mathcal{C}, k\rangle$. The MAX-SAT problem is NP-complete [48].

Definition 2 [D-UDCP] Given the network of MRs as a graph $G(\mathcal{B} \cup \mathcal{G}, E)$, where $E$ is the set of connectivity edges between MRs, and the channel availability at each $\operatorname{MR}\left(\mathcal{L}_{i} \forall i \in \mathcal{B} \cup \mathcal{G}\right)$. For an integer $u \leq|\mathcal{B}|$, is there a receiver-based channel assignment that guarantees both upstream and downstream connectivity for at least $u$ non-gateway MRs? Let us denote this problem as D-UDCP $\left\langle\mathcal{B}, \mathcal{G}, E, \mathcal{L}_{i} \forall i \in \mathcal{B} \cup \mathcal{G}, u\right\rangle$.

Lemma 1: D-UDCP $\in$ NP.

Proof: Let $\omega=|\mathcal{B}|+|\mathcal{G}|+1$. Then, after adding a hypothetical node to the graph, the total number of directed edges, which result from the receiver-based channel assignment, in the graph is upper bounded by $\omega(\omega-1)$. The following verifier is linear in the size of the graph (number of vertices plus number of edges).

Verifier D-UDCP $\left\langle\mathcal{B}, \mathcal{G}, E, \mathcal{L}_{i} \forall i \in \mathcal{B} \cup \mathcal{G}, u\right\rangle$ :

1) Add a hypothetical node $s$ to the graph and make it bi-connected with all nodes in $\mathcal{G}$.

2) Run a Depth-First-Search (DFS) algorithm starting from $s$ and mark all reachable nodes as downstream connected. $O\left(\omega+\omega(\omega-1)=O\left(\omega^{2}\right)\right.$

3) Reverse all edges in the graph.

4) Run a Depth-First-Search (DFS) algorithm starting from $s$ and mark all reachable nodes as upstream connected. $O(\omega+$ $\omega(\omega-1)=O\left(\omega^{2}\right)$

5) If the total number of nodes from $\mathcal{B}$ that are upstream AND downstream connected is greater than or equal to $u$ then accept, otherwise reject.

\section{Lemma 2: MAX-SAT $\leq_{p}$ D-UDCP.}

Proof: Any instance of the MAX-SAT problem, say MAX-SAT $\langle\mathcal{X}, \mathcal{C}, k\rangle$, can be mapped into an instance of the D-UDCP problem as follows:

- Add a gateway MR $g$ to $\mathcal{G}$ and define its set of available channels to be $\{0,1\}$.

- Add a non-gateway MR for each variable, and a non-gateway MR for each clause to $\mathcal{B}$ with the set of available channels be $\{0,1\}$ in both cases. Extend an edge between the gateway MR and every non-gateway MR that represents a variable, and add these edges to $E$.

- For each non-gateway MR that represents a clause, say $C_{i}$, add three non-gateway MRs $n_{i}, b_{i}$, and $p_{i}$ which we call auxiliary MRs to $\mathcal{B}$. Then, extend edges between MR $n_{i}$ (resp. $p_{i}$ ) and the MR that represents $C_{i}$ as well as those which represent the variables that appear in a negative (resp. positive) form in the clause $C_{i}$ and add them to $E$. Finally, extend edges between $b_{i}$ and both $n_{i}$ and $p_{i}$, and also add them to $E$. The sets of available channels for $n_{i}, b_{i}$, and $p_{i}$ are $\{0\}$, $\{0,1\}$, and $\{1\}$ respectively. We refer to set $\left\{n_{i}, b_{i}, p_{i}\right\}$ as the auxiliary set of the MRs that represents clause $C_{i}$.

- $u=4 \times k+N$, where $N=|\mathcal{X}|$.

The above mapping can be rewritten as follows:

$\mathcal{G}=\{g\}$

$\mathcal{B}=\left\{x_{i}: x_{i} \in \mathcal{X}\right\} \cup\left\{C_{i}, n_{i}, b_{i}, p_{i}: C_{i} \in \mathcal{C}\right\}$

$E=\left\{\left(g, x_{i}\right): x_{i} \in \mathcal{X}\right\} \cup\left\{\left(n_{i}, b_{i}\right),\left(b_{i}, p_{i}\right),\left(p_{i}, C_{i}\right),\left(n_{i}, C_{i}\right): C_{i} \in \mathcal{C}\right\} \cup\left\{\left(n_{i}, x_{j}\right): \bar{x}_{j} \in \mathcal{C}_{i}\right\} \cup\left\{\left(p_{i}, x_{j}\right): x_{j} \in \mathcal{C}_{i}\right\}$

$\mathcal{L}_{n_{i}}=\{0\}, \mathcal{L}_{p_{i}}=\{1\}, \mathcal{L}_{b_{i}}=\mathcal{L}_{x_{i}}=\mathcal{L}_{C_{i}}=\{0,1\}$

Note that all edges in $E$ are now undirected. However, the solution will return directed edges due to the receiver-based nature of the channel assignment.

An example of this mapping process is shown in Figure 16. Let an MR that represents a clause be denoted by CMR, an MR that represents a variable be denoted by VMR, and an auxiliary MR be denoted by AMR. According to the mapping procedure described above:

1) All VMRs are always upstream-and-downstream connected regardless of what channels are allocated to those VMRs and the gateway MR. 
2) If at least one AMR from the auxiliary set of a CMR is upstream-and-downstream connected with the gateway, then there exists a channel allocation solution that guarantees all other AMRs of that set as well as the CMR to be upstreamand-downstream connected as shown in Figure 17. It follows that if more than one AMR are upstream-downstream connected, then the CMR and all AMRs in its auxiliary set are also upstream-and-downstream connected. It also follows that if neither the $n_{i}$ AMR nor the $p_{i}$ AMR is upstream-and-downstream connected, then the CMR $C_{i}$ and all the AMRs in its auxiliary set are not upstream-and-downstream connected. In other words, a CMR and its auxiliary set are either upstream-and-downstream connected together, or not upstream-and-downstream together.

Suppose MAX-SAT $\langle\mathcal{X}, \mathcal{C}, k\rangle$ has a boolean assignment $\mathcal{X}^{*}$, where $\mathcal{X}^{*}(i) \in\{T R U E, F A L S E\}$ is the boolean value of $x_{i}$, that satisfies the subset of clauses $\mathcal{C}^{*} \subseteq \mathcal{C}$ such that $\left|\mathcal{C}^{*}\right| \geq k$. Then, there must be a receiver-based channel assignment that guarantees both upstream and downstream connectivity for at least $u=4 \times k+N$ non-gateway MRs. This can be easily proven as follows. Allocate either channel 0 or 1 to the gateway MR $g$. Then, for each variable $x_{i}$, if $\mathcal{X}^{*}(i)=$ TRUE (resp. $=$ FALSE) allocate channel 1 (resp. 0) to VMR $x_{i}$. This guarantees upstream and downstream connectivity to all the $N$ VMRs. Then, allocate channel 0 to all $n_{i}$ 's and channel 1 to all $p_{i}$ 's. For each $C_{i} \in \mathcal{C}^{*}$, one of the following must hold:

- At least one variable, say $x_{j}$, that appeared in a positive form in $C_{i}$ has $\mathcal{X}^{*}(i)=\mathrm{TRUE}$. Therefore, VMR $x_{j}$, which is (by construction) a neighbor of $p_{i}$, must be allocated channel 1 . As $p_{i}$ is also allocated channel 1 , and VMR $x_{j}$ is upstream-and-downstream connected, then $p_{i}$ is also upstream-and-downstream connected. As explained in Figure 17, there exists a receiver-based channel allocation that makes CMR $C_{i}$ and its auxiliary set all upstream-and-downstream connected.

- At least one variable, say $x_{j}$, that appeared in a negative form in $C_{i}$ has $\mathcal{X}^{*}(i)=$ FALSE. Therefore, VMR $x_{j}$, which is (by construction) a neighbor of $n_{i}$, must be allocated channel 0 . As $n_{i}$ is also allocated channel 0 , and VMR $x_{j}$ is upstream-and-downstream connected, then $n_{i}$ is also upstream-and-downstream connected. As explained in Figure 17, there exists a receiver-based channel allocation that makes CMR $C_{i}$ and its auxiliary set all upstream-and-downstream connected.

As the auxiliary set of each CMR is of size 3, the total number of upstream-and-downstream connected non-gateway MRs is $N+\left|\mathcal{C}^{*}\right|+3 \times\left|\mathcal{C}^{*}\right| \geq N+4 \times k$. Conversely, suppose $\mathrm{D}$ - $\mathrm{UDCP}\langle\mathcal{B}, \mathcal{G}, E, u=4 \times k+N\rangle$ has a receiver-based channel assignment $\mathcal{L}^{*}$, where $\mathcal{L}^{*}(i) \in\{0,1\}$ is the channel allocated to MR $i$. Then, there is a boolean assignment to $\mathcal{X}$ such that at least $k$ clauses from $\mathcal{C}$ are satisfied. Simply, for each VMR that is assigned channel 0 (resp. 1), assign the variable it represents a value of FALSE (resp. TRUE). Then, the same arguments made above can be used to show that at least $k$ clauses will be satisfied. If a CMR $C_{i}$ is upstream-and-downstream, then at least one of the two AMRs $p_{i}$ and $n_{i}$ must also be upstreamand-downstream connected. If $p_{i}\left(\right.$ resp. $\left.n_{i}\right)$ is upstream-and-downstream connected, then there must be at least one VMR that represents a variable, say $x_{j}$, that appeared in a positive (resp. negative) form in clause $C_{i}$ which is assigned channel 1 (resp. channel 0). Given the channel-to-boolean mapping described earlier, clause $C_{i}$ is satisfied in the MAX-SAT $\langle\mathcal{X}, \mathcal{C}, k\rangle$ instance. Therefore, the MAX-SAT $\langle\mathcal{X}, \mathcal{C}, k\rangle$ instance has at least $\frac{u-N}{4} \geq k$ satisfied clauses.

This concludes the proof that D-UDCP is NP-complete. As UDCP is the optimization version of D-UDCP, then UDCP is NP-hard. 
C $_{1}^{\left\{x_{1} \vee \bar{x}_{2} \vee x_{3} \vee x_{4}\right\} \wedge\left\{\bar{x}_{1} \vee x_{2} \vee \bar{x}_{3} \vee \bar{x}_{4}\right\} \wedge\left\{x_{1} \vee x_{2} \vee x_{3} \vee x_{4}\right\}} C_{2} \longmapsto C_{3}$

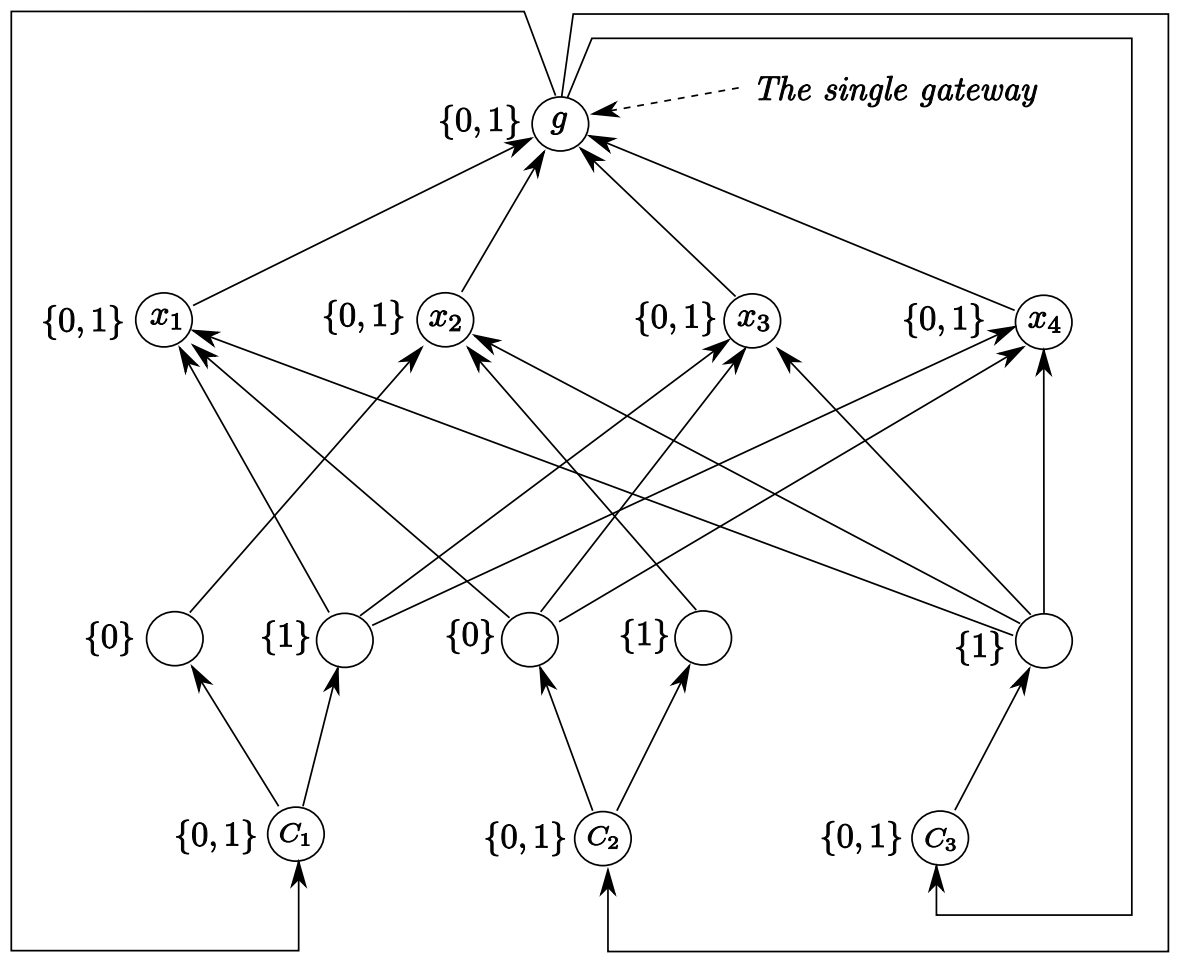

Fig. 16. Mapping a maximum $K-S A T$ problem into an UDCP problem.

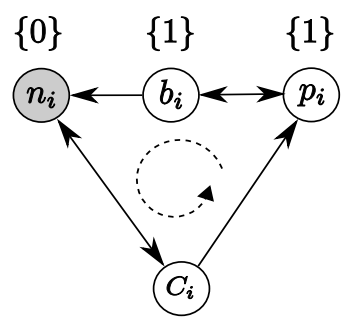

$\{0\}$

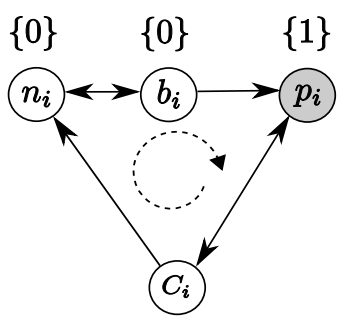

$\{1\}$

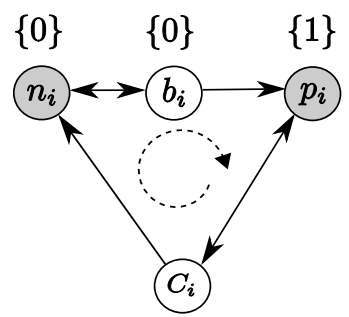

$\{1\}$

CASE $(a)$

CASE (b)

CASE $(c)$

Fig. 17. A receiver-based channel allocation that can make the CMR $i$ and its auxiliary set upstream-and-downstream connected given that either $p_{i}, n_{i}$, or both are upstream-and-downstream connected (gray colored). 